\title{
Metallalkenyl, Metallacyclopropene, or Metallallylcarbenoid? Ru-Catalyzed Annulation between Benzoic Acid and Alkyne
}

Jingxing Jiang, ${ }^{\dagger}$ Honghu Liu, ${ }^{\dagger}$ Lili Cao, ${ }^{+}$Cunyuan Zhao, ${ }^{+}$Yan Liu, ${ }^{*,+}$ Lutz Ackermann,,,$\S$ and Zhuofeng $\mathrm{Ke}^{*,+}$

${ }^{+}$School of Materials Science \& Engineering, PCFM Lab, School of Chemistry, Sun Yat-sen University, Guangzhou 510275, P. R. China

'School of Chemical Engineering and Light Industry, Guangdong University of Technology, Guangzhou 510006, P. R. China

Institut für Organische und Biomolekulare Chemie, Georg-August-Universität, Tammannstrasse 2, 37077 Göttingen, Germany

Email: yanliu@gdut.edu.cn; ackermann@chemic.uni-goettingen.de;kezhf3@mail.sysu.edu.cn

\section{Content}

1. Computational Method and Details.

2. Other Essential Pathways in Ru-Catalyzed Annulation between Benzoic Acid and Alkyne

3. Selected Structures of Ru-catalyzed Annulation between Benzoic Acid and 2-Butyne

4. Other Information of Ru-catalyzed Annulation between Benzoic Acid and 2-Butyne

5. Original Energetic Data and Thermal Corrections of All Species 


\section{Computational Method and Details.}

All calculations were performed with the Gaussian 09 software package. ${ }^{1}$ Density functional theory (DFT) calculations using the B3LYP functional ${ }^{2}$ were used to locate all the stationary points involved. The SDD pseudopotential and basis set $^{3}$ is applied for the ruthenium center whereas other non-metal elements use 6-31G(d,p) basis set. Frequency calculations at the same level of theory for the geometry optimizations confirm that each stationary point is a transition state or an energy minimum structure. We confirmed TS structures by intrinsic reaction coordinate (IRC) calculations ${ }^{4}$ to connect the correct reactant/product and intermediates. These structures are optimized by a self-consistent reaction field (SCRF) using the $\mathrm{SMD}^{5}$ implicit solvent model. The parameter for methanol $(\varepsilon=32.613)$ are chosen to avoid the complicated definition of custom parameterized tert-amyl alcohol (not defined in Gaussian 09 program). Moreover, the methanol solvent is also experimentally available in the annulation of benzoic acid with alkyne by $\mathrm{Ru}$ catalyst.

To get better accuracy of energies, we recalculated the single-point energies of optimized structures with a weak interaction involved M06 functional ${ }^{6}$, a larger basis set $(6-311++\mathrm{G}(2 \mathrm{~d}, 2 \mathrm{p})$ for non-metal elements and SDD basis set with extra $f$-polarization ${ }^{7} /$ outer $p^{8}$ functions for $\mathrm{Ru}$ ) and SMD implicit solvent environment. In this multicomponent system, entropies are overestimated due to the thermal corrections are based on the ideal gas model which ignores the solvent suppression on the translational and rotational freedoms of solutes. ${ }^{9}$ We used an empirical approach to correct the entropies in solution: only vibrational contribution of entropies are reserved ${ }^{10}$ in a reaction at $318.15 \mathrm{~K}$ and 1 bar (Table S1).

In addition, for comprehensively analyzing the origin of the mechanistic and regio- selectivity, NPA charge calculation was carried out by using NBO 5.0.11 The orbital compositions (Becke method) are summarized by using the Multiwfn analyzer. ${ }^{12}$ Fragment distortion energies were computed at the M06/6-311++G(2d,2p)-SDD(d,f)/SMD level using the B3LYP/6-31G(d,p)-SDD/SMD geometries.

\section{References}

1. Frisch, M. J.; Trucks, G. W.; Schlegel, H. B.; Scuseria, G. E.; Robb, M. A.; Cheeseman, J. R.; Scalmani, G.; Barone, V.; B. Mennucci; Petersson, G. A.; Nakatsuji, H.; Caricato, M.; Li, X.; Hratchian, H. P.; Izmaylov, A. F.; Bloino, J.; Zheng, G.; Sonnenberg, J. L.; Hada, M.; Ehara, M.; Toyota, K.; Fukuda, R.; Hasegawa, J.; Ishida, M.; Nakajima, T.; Honda, Y.; Kitao, O.; Nakai, H.; Vreven, T.; J. A. Montgomery, J.; Peralta, J. E.; Ogliaro, F.; Bearpark, M.; J. J. Heyd, E. Brothers; Kudin, K. N.; Staroverov, V. N.; Kobayashi, R.; Normand, J.; Raghavachari, K.; Rendell, A.; Burant, J. C.; Iyengar, S. S.; Tomasi, J.; Cossi, M.; Rega, N.; Millam, J. M.; Klene, M.; Knox, J. E.; Cross, J. B.; Bakken, V.; Adamo, C.; Jaramillo, J.; Gomperts, R.; Stratmann, R. E.; Yazyev, O.; Austin, A. J.; Cammi, R.; Pomelli, C.; Ochterski, J. W.; Martin, R. L.; Morokuma, K.; Zakrzewski, V. G.; Voth, G. A.; Salvador, P.; Dannenberg, J. J.; Dapprich, S.; Daniels, A. D.; Farkas, O.; Foresman, J. B.; Ortiz, J. V.; Cioslowski, J.; Fox, D. J.; Gaussian 09, Revision D.01; Gaussian, Inc.: Wallingford, CT, 2009.

2. (a) Lee C.; W. Yang W.; Parr R. G. Development of the Colle-Salvetti correlation-energy formula into a functional of the electron density. Phys. Rev. B: Condens. Matter, 1988, 37, 785- 
789. (b) Becke A. D. Density-functional thermochemistry. III. The role of exact exchange. J. Chem. Phys., 1993, 98, 5648-5652.

3. Andrae D.; Häußermann U.; Dolg M.; Stoll H.; Preuß H. Energy-adjustedab initio pseudopotentials for the second and third row transition elements Theor. Chim. Acta, 1990, 77, 123-141.

4. (a) Fukui K. Formulation of the reaction coordinate. J. Phys. Chem., 1970, 74, 4161-4163. (b) Fukui K., The path of chemical reactions - the IRC approach. Acc. Chem. Res., 1981, 14, 363-368.

5. Marenich A. V.; Cramer C. J.; Truhlar D. G. Universal solvation model based on solute electron density and on a continuum model of the solvent defined by the bulk dielectric constant and atomic surface tensions. J. Phys. Chem. B, 2009, 113, 6378-6396.

6. Zhao, Y.; Truhlar, D. G., The M06 suite of density functionals for main group thermochemistry, thermochemical kinetics, noncovalent interactions, excited states, and transition elements: two new functionals and systematic testing of four M06-class functionals and 12 other functionals. Theor. Chem. Acc. 2007, 120, 215-241.

7. Couty M.; Hall M. B. Basis sets for transition metals: Optimized outer $\mathrm{p}$ functions. J. Comput. Chem., 1996, 17, 1359-1370.

8. Ehlers A. W.; Böhme M.; Dapprich S.; Gobbi A.; Höllwarth A.; Jonas V.; Köhler K. F.; Stegmann R.; Veldkamp A.; Frenking G. A set of f-polarization functions for pseudo-potential basis sets of the transition metals Sc-Cu, Y-Ag and La-Au. Chem. Phys. Lett., 1993, 208, 111-114.

9. (a) Wertz, D. H. Relationship between the gas-phase entropies of molecules and their entropies of solvation in water and 1-octanol. J. Am. Chem. Soc. 1980, 102, 5316. (b) Leung, B. O.; Reid, D. L.; Armstrong, D. A.; Rauk, A. Entropies in Solution from Entropies in the Gas Phase. J. Phys. Chem. A 2004, 108, 2720.

10. (a) Sumimoto M.; Iwane N.; Takahama T.; Sakaki S. Theoretical Study of Trans-metalation Process in Palladium-Catalyzed Borylation of Iodobenzene with Diboron. J. Am. Chem. Soc., 2004, 126, 10457-10471. (b) Sasaki S.; Takayama T.; Sumimoto M.; Sugimoto M. Theoretical Study of the Cp2Zr-Catalyzed Hydrosilylation of Ethylene. Reaction Mechanism Including New $\sigma$-Bond Activation. J. Am. Chem. Soc., 2004, 126, 3332-3348.

11. Glendening E. D.; Badenhoop J. K.; Reed A. E.; Carpenter J. E.; Bohmann J. A.; Morales C. M.; Weinhold F. NBO 5.0, Theoretical Chemistry Institute, University of Wisconsin, Madison, 2001.

12. Lu T.; Chen F. Multiwfn: a multifunctional wavefunction analyzer. J. Comput. Chem., 2012, 33, 580-592. 
2. Other Essential Pathways in Ru-Catalyzed Annulation between Benzoic Acid and Alkyne
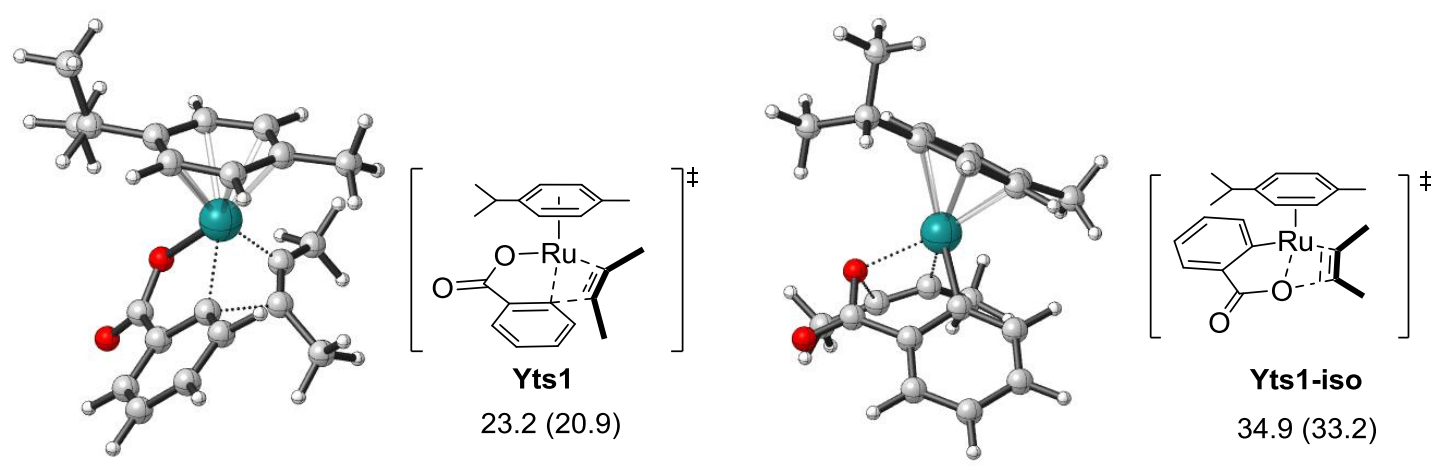

Figure $\mathrm{S1}$. $\mathrm{Ru}-\mathrm{C}$ bond vs $\mathrm{Ru}-\mathrm{O}$ bond: alkyne insertion. Free energies (Enthalpies) are given in $\mathrm{kcal} / \mathrm{mol}$.

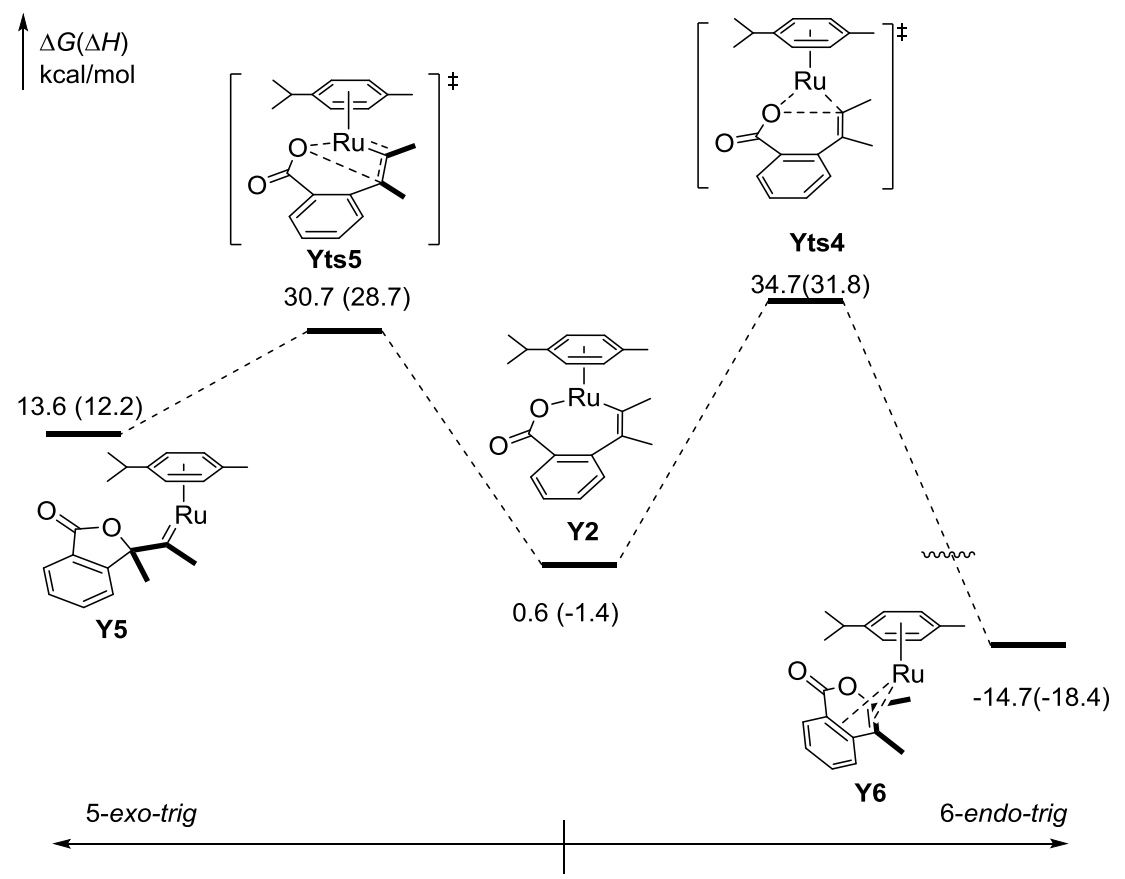

Figure S2. Potential energy profiles of annulation from metallalkenyl intermediate Y3. 
3. Selected Structures of Ru-catalyzed Annulation between Benzoic Acid and 2-Butyne
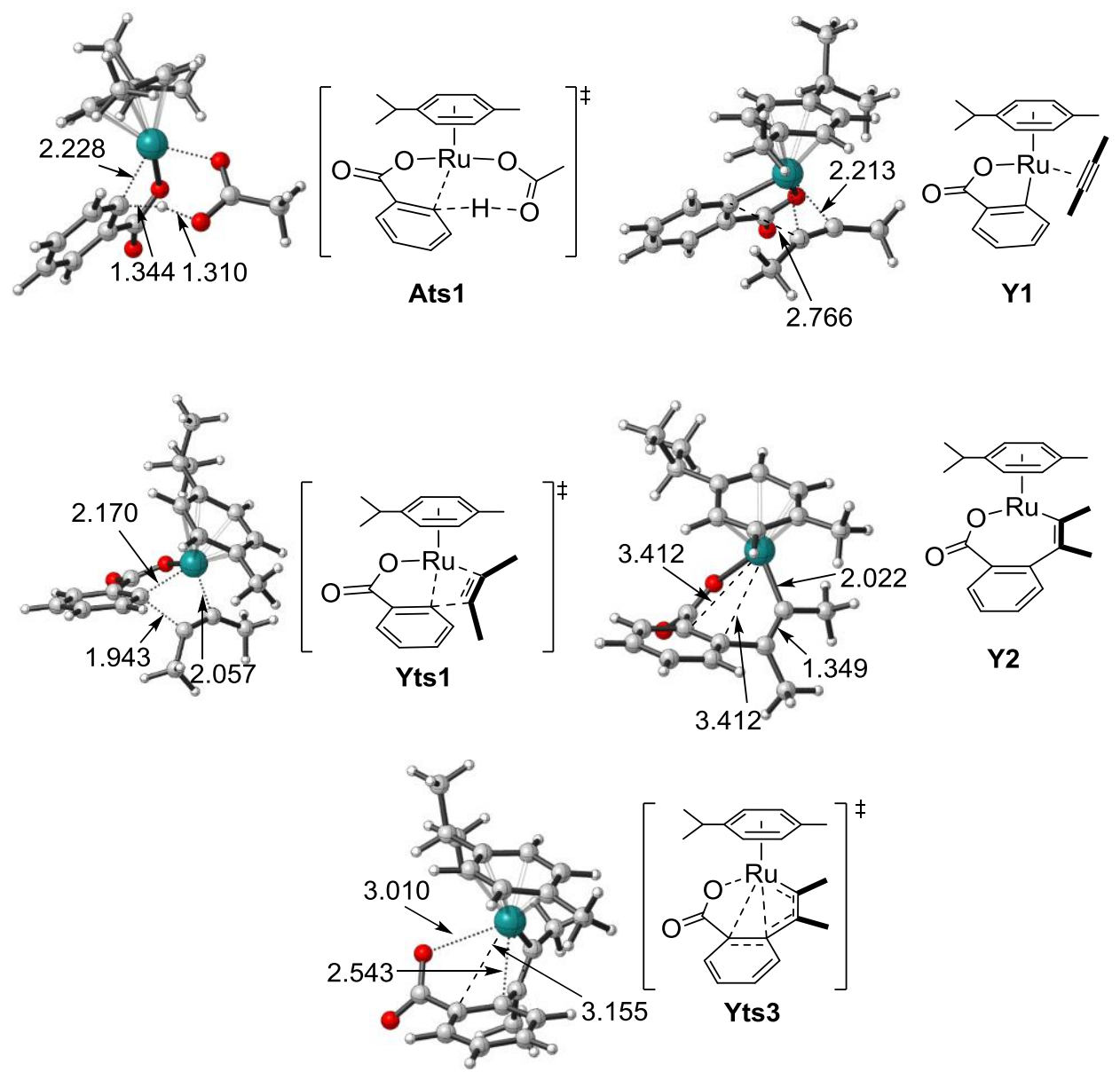

Yts3

Figure S3. DFT-optimized structures of selected intermediates and transition states of the Ru-catalyzed annulation between benzoic acid and 2-butyne. Bond distances are given in $\AA$. 
4. Other Information of Ru-catalyzed Annulation between Benzoic Acid and 2-Butyne
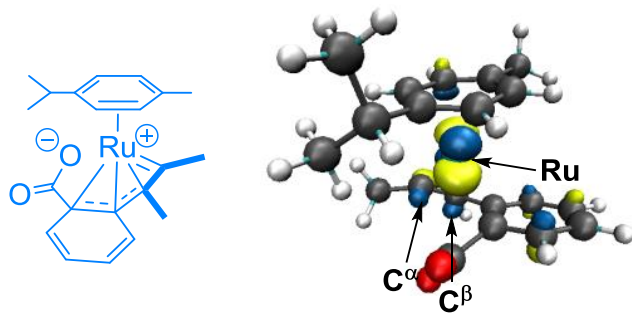

Orbital Composition

$\mathrm{Ru}: \mathbf{4 0 . 6 \%}$

$C^{\alpha}: 6.13 \% C^{\beta}: 8.08 \%$

Figure S4. LUMO in metallallylcarbenoid intermediates Y4. (Isovalue $=0.07$ )

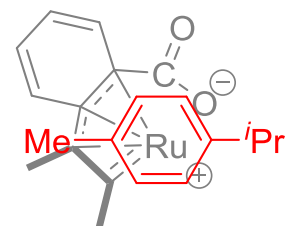

0.0

Original

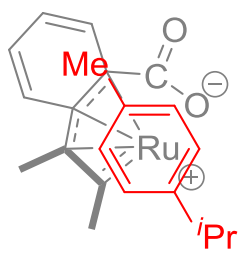

0.3

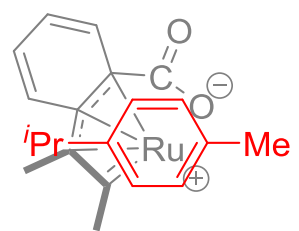

0.6

A

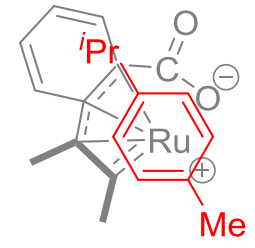

1.0

C

Figure S5. Conformations of Y4. Free energies are given in $\mathrm{kcal} / \mathrm{mol}$. 


\section{Original Energetic Data and Thermal Corrections of All Species}

Table S1. Original energetic data and thermal corrections of all species. Energies are in $\mathrm{kcal} / \mathrm{mol}$, entropy values are in $\mathrm{cal} /(\mathrm{mol} \bullet \mathrm{K})$ others are in Hartree.

\begin{tabular}{|c|c|c|c|c|c|c|}
\hline Species & $\begin{array}{c}\mathrm{H} \\
\text { correction }\end{array}$ & $\begin{array}{c}\mathrm{G} \\
\text { correction }\end{array}$ & $\begin{array}{c}\text { Solvated } \\
\text { Energy }\end{array}$ & $\begin{array}{c}\text { Vibrational } \\
\text { Entropy }\end{array}$ & $\Delta \mathrm{G}^{, 1}$ & $\Delta \mathrm{G}^{, 2}$ \\
\hline$(\mathrm{HOAc})_{2}$ & 0.135603 & 0.089089 & -458.110718 & 28.8 & I & I \\
\hline 2-butyne & 0.090813 & 0.056058 & -155.901392 & 11.9 & I & I \\
\hline A1 & 0.396649 & 0.314054 & -1132.807068 & 94.7 & 0.0 & 0.0 \\
\hline A1' & 0.397374 & 0.315613 & -1132.784129 & 93.6 & 15.4 & 15.1 \\
\hline Ats1 & 0.390900 & 0.312581 & -1132.761454 & 86.2 & 27.7 & 26.4 \\
\hline A2 & 0.397513 & 0.318853 & -1132.786953 & 86.8 & 15.6 & 14.4 \\
\hline Y1 & 0.420325 & 0.338836 & -1059.644002 & 93.0 & 14.0 & 10.1 \\
\hline Yts1 & 0.418757 & 0.341061 & -1059.618877 & 85.1 & 31.2 & 26.1 \\
\hline Y2 & 0.421090 & 0.342925 & -1059.656819 & 85.8 & 8.6 & 3.6 \\
\hline Y2' & 0.491051 & 0.398677 & -1288.716062 & 114.5 & 13.2 & 5.3 \\
\hline Yts2 & 0.419632 & 0.343185 & -1059.641935 & 82.2 & 18.1 & 12.5 \\
\hline Y3 & 0.420686 & 0.341379 & -1059.643355 & 84.0 & -2.0 & 11.4 \\
\hline Yts3 & 0.419837 & 0.341447 & -1059.645961 & 86.5 & 14.4 & 9.5 \\
\hline Y4 & 0.420910 & 0.342207 & -1059.661656 & 87.3 & 5.1 & 0.2 \\
\hline Yts4' & 0.488753 & 0.398939 & -1288.659658 & 109.1 & 48.7 & 40.1 \\
\hline Y6' & 0.489971 & 0.399529 & -1288.676059 & 110.4 & 38.8 & 30.3 \\
\hline Yts5' & 0.489096 & 0.399629 & -1288.672018 & 108.7 & 41.4 & 32.6 \\
\hline Y5 & 0.490441 & 0.400421 & -1288.681309 & 109.5 & 36.1 & 27.5 \\
\hline Yts4 & 0.419044 & 0.342261 & -1059.601868 & 84.0 & 42.6 & 37.2 \\
\hline Y6 & 0.421473 & 0.346088 & -1059.684214 & 80.4 & -6.6 & -12.5 \\
\hline Yts5 & 0.418701 & 0.339793 & -1059.605662 & 87.3 & 22.7 & 33.9 \\
\hline Y5 & 0.420592 & 0.341636 & -1059.634639 & 87.7 & 3.6 & 16.9 \\
\hline Yts6 & 0.419325 & 0.342028 & -1059.627238 & 84.0 & 10.5 & 21.3 \\
\hline Yts7 & 0.419565 & 0.342435 & -1059.657211 & 84.1 & 8.0 & 2.7 \\
\hline Yts8 & 0.418868 & 0.339487 & -1059.625281 & 88.6 & 26.2 & 21.6 \\
\hline Yts1-iso & 0.418325 & 0.339789 & -1059.598882 & 86.8 & 42.6 & 38.1 \\
\hline
\end{tabular}

1. In this column, we did not correct the entropies, i.e., translational, rotational and vibrational contributions are reserved.

2. In this column, we estimated the total entropy in solution at $50 \%$. Please see:

(1) Yu Z. X. and Houk K. N., Intramolecular 1,3-Dipolar Ene Reactions of Nitrile Oxides Occur by Stepwise 1,1-Cycloaddition/Retro-Ene Mechanisms, J. Am. Chem. Soc. 2003, 125, 13825-13830.

(2) Liang Y., Liu S., Xia Y., Li Y., Yu Z. X., Chem. -Eur. J. 2008, 14, 4361-4373.

(3) Plata R. E., Singleton D. A., A Case Study of the Mechanism of Alcohol-Mediated Morita BaylisHillman Reactions. The Importance of Experimental Observations. J. Am. Chem. Soc. 2015, 137, 3811-3826.

Herein, if we used these two entropic correction patterns, the alkyne migratory insertion transition state Yts 1 
is calculated to be 31.2 and $26.1 \mathrm{kcal} / \mathrm{mol}$, being higher than or close to Ats1 $(27.7$ or $26.4 \mathrm{kcal} / \mathrm{mol}$ for pattern 1 or 2, respectively). Moreover, the metallallylcarbenoid mechanism to 6-endo-trig product is preferred over any other pathways. In another word, our entropic correction did not change the mechanistic and regio- selectivities. Therefore, the entropic correction of translational/rotational contributions removal agrees with the experimental observation and does not change our conclusion.

Table S2. Original energetic data and free energy corrections of Y4 conformations. Energies are in $\mathrm{kcal} / \mathrm{mol}$, others are in Hartree.

\begin{tabular}{ccccc}
\hline Conformations & H correction & G correction & Solvated Energy & Vibrational Entropy \\
\hline Y4 (Original) & 0.420910 & 0.342207 & -1059.661656 & 87.3 \\
A & 0.420793 & 0.341538 & -1059.660052 & 88.5 \\
B & 0.420662 & 0.341914 & -1059.660866 & 87.4 \\
C & 0.420608 & 0.341160 & -1059.629030 & 88.9 \\
\hline
\end{tabular}


6. Origin XYZ coordinates of All Species

$(\mathrm{HOAc})_{2}$

$\begin{array}{llll}\mathrm{C} & 1.9253400 & 0.0584110 & -0.0001320 \\ \mathrm{O} & 1.3820990 & -1.1460120 & -0.0015730 \\ \mathrm{C} & 3.4255040 & 0.0457820 & -0.0000250 \\ \mathrm{H} & 3.7877370 & -0.4951430 & -0.8801380 \\ \mathrm{H} & 3.8117750 & 1.0648150 & -0.0037980 \\ \mathrm{H} & 3.7869670 & -0.4878620 & 0.8849320 \\ \mathrm{O} & 1.2622130 & 1.1019260 & 0.0013710 \\ \mathrm{H} & -0.3759020 & 1.0852550 & -0.0002040 \\ \mathrm{O} & -1.3820980 & 1.1460280 & -0.0012470 \\ \mathrm{C} & -1.9254200 & -0.0583950 & 0.0000480 \\ \mathrm{O} & -1.2621090 & -1.1017900 & 0.0017410 \\ \mathrm{C} & -3.4255440 & -0.0459030 & -0.0002440 \\ \mathrm{H} & -3.7874670 & 0.4904500 & 0.8828620 \\ \mathrm{H} & -3.7873910 & 0.4922850 & -0.8822180 \\ \mathrm{H} & -3.8117330 & -1.0649710 & -0.0011850 \\ \mathrm{H} & 0.3758880 & -1.0854080 & -0.0004730\end{array}$

\section{2-butyne}

C $\quad-0.6058640$

C $\quad 0.6058620$

C $\quad-2.0676310$

H $\quad-2.4668930$

H $\quad-2.4658760$

H $\quad-2.4665880$

C 2.0676310

H 2.4667910

H 2.4663570

H 2.4662110

\section{A1}

$\mathrm{Ru} \quad-0.6833740$

C $\quad-2.8487430$

C $\quad-2.6724560$

C -1.8847360

C -1.3309980

C -1.4972400

C $\quad-2.2759540$

$\mathrm{H} \quad-3.3488090$

H $\quad-1.6426230$
$-0.0007250$

$-0.0000970$

0.0009040

$-0.0000110$

0.0000600

0.2477780

0.7344630

$-0.9814450$

0.0000950

$-0.7327360$

$-0.2492980$

0.9823620

0.0001770

0.9906530

$-0.7098400$

$-0.2815230$

$-0.0002840$

0.7108430

$-0.9905560$

0.2792850

0.2873990

$-0.2037290$

0.0740960

0.4759140

$-0.5766700$

0.7804460

1.6495200

1.0062720

$-0.0565700$

2.4131510

1.9790290

$-1.4002810$

0.8039700

$-1.6495750$

$-0.8615860$

1.9250570

$-0.7992680$

2.0285700

\begin{tabular}{|c|c|c|c|}
\hline $\mathrm{H}$ & -0.6930280 & 3.2619300 & 0.1634610 \\
\hline $\mathrm{H}$ & -2.3652850 & 0.4284390 & -2.6622740 \\
\hline C & -3.2636970 & -0.2744030 & 1.9631890 \\
\hline I & -2.5558740 & -0.1530600 & 2.7932500 \\
\hline C & -0.8231020 & 2.6950080 & -2.5345870 \\
\hline $\mathrm{H}$ & 0.0539360 & 3.2485100 & -2.1902760 \\
\hline $\mathrm{H}$ & -0.5196740 & 1.9942840 & -3.3171030 \\
\hline $\mathrm{H}$ & -1.5240200 & 3.4117530 & -2.9802800 \\
\hline $\mathrm{C}$ & -4.5898620 & 0.3947060 & 2.3775900 \\
\hline $\mathrm{H}$ & -4.9956630 & -0.0932480 & 3.2702990 \\
\hline $\mathrm{H}$ & -4.4503950 & 1.4565710 & 2.6065280 \\
\hline $\mathrm{H}$ & -5.3369010 & 0.3145670 & 1.5791370 \\
\hline $\mathrm{C}$ & -3.4570420 & -1.7765990 & 1.7213630 \\
\hline $\mathrm{H}$ & -2.5273000 & -2.2580710 & 1.4030750 \\
\hline $\mathrm{H}$ & -3.7877170 & -2.2573710 & 2.6477490 \\
\hline $\mathrm{H}$ & -4.2226470 & -1.9731200 & 0.9624810 \\
\hline $\mathrm{C}$ & -0.4136740 & -2.5502230 & -1.2226210 \\
\hline $\mathrm{O}$ & -0.7957240 & -2.1768800 & -2.3454010 \\
\hline $\mathrm{O}$ & -0.3015490 & -1.7857890 & -0.1778390 \\
\hline $\mathrm{C}$ & 0.0086970 & -3.9904430 & -0.9851530 \\
\hline $\mathrm{H}$ & 1.1036750 & -4.0452750 & -0.9923580 \\
\hline $\mathrm{H}$ & -0.3331340 & -4.3463600 & -0.0095180 \\
\hline $\mathrm{H}$ & -0.3767470 & -4.6386250 & -1.7748200 \\
\hline $\mathrm{C}$ & 3.8274280 & 0.1458130 & 1.7432650 \\
\hline $\mathrm{C}$ & 5.2012980 & 0.1143280 & 1.9736960 \\
\hline C & 6.0919400 & 0.1217370 & 0.8958620 \\
\hline $\mathrm{C}$ & 5.6076080 & 0.1597940 & -0.4153240 \\
\hline $\mathrm{C}$ & 4.2345310 & 0.1930030 & -0.6514650 \\
\hline $\mathrm{C}$ & 3.3378540 & 0.1854790 & 0.4283750 \\
\hline $\mathrm{H}$ & 3.1305020 & 0.1448190 & 2.5749700 \\
\hline $\mathrm{H}$ & 5.5779360 & 0.0865870 & 2.9920020 \\
\hline $\mathrm{H}$ & 7.1628470 & 0.0998700 & 1.0777570 \\
\hline $\mathrm{H}$ & 6.3004470 & 0.1659270 & -1.2517300 \\
\hline $\mathrm{C}$ & 1.8773890 & 0.2471580 & 0.1824660 \\
\hline $\mathrm{O}$ & 1.4044910 & 0.3553550 & -0.9994790 \\
\hline $\mathrm{O}$ & 1.0417430 & 0.2136450 & 1.1511060 \\
\hline & 3.8517880 & 0.2259690 & -1.6663320 \\
\hline
\end{tabular}

\section{Ats 1}

$\mathrm{Ru} \quad-0.2903450$

0.3719680

0.1004280

C $\quad-2.4795070$

0.8985110

0.4735690 


\begin{tabular}{|c|c|c|c|c|c|c|c|}
\hline$C$ & 0 & 30 & 0 & \multirow{2}{*}{ A } & & & \\
\hline $\mathrm{C}$ & -1.4150730 & -1.1540700 & 1.2538220 & & & & \\
\hline $\mathrm{C}$ & -0.6193490 & -0.4019170 & 2.1476060 & $\mathrm{Ru}$ & -1.0059570 & 0.5204860 & 0.06789 \\
\hline$C$ & -0.7288510 & 1.0250640 & 2413080 & $C$ & -3.0285240 & -0.3597240 & -0.05456 \\
\hline C & -1.6352070 & 6480400 & 0 & 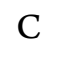 & -2.1276860 & -1.3960320 & 0.3722370 \\
\hline $\mathrm{H}$ & -3.1355650 & 1.4353090 & 0.2007420 & $\mathrm{C}$ & -1.3218760 & -1.1257710 & 1.5057420 \\
\hline $\mathrm{H}$ & -1.2747540 & -2.2276630 & 1.1854820 & $+>$ & -1.3944320 & 0.1224110 & 2.1984330 \\
\hline $\mathrm{H}$ & 0.1267260 & -0.9 & 0 & $\mathrm{C}$ & -2 . & 0 & 750 \\
\hline $\mathrm{H}$ & -1.6754550 & 2.73 & 040 & $C$ & -3.12 & 0.8 & 8290 \\
\hline $\mathrm{C}$ & -3.2204360 & -1.3535880 & -0.5345060 & $\mathrm{H}$ & -3.6008560 & -0.4890710 & -0.966217 \\
\hline $\mathrm{H}$ & -2.5833710 & -2.1859750 & -0.8585780 & $\mathrm{H}$ & -0.5683630 & -1.8485010 & 1.8013250 \\
\hline $\mathrm{C}$ & 0.0708760 & 1.81 & 3.23 & 1 & -0.7 & 0.32 & 3.0 \\
\hline $\mathrm{H}$ & 0.9978860 & 1.30 & 0 & I & 80 & 0 & 4410 \\
\hline $\mathrm{H}$ & 0.3114650 & 2.8131110 & 2.8531220 & $\mathrm{C}$ & -2.0336970 & -2.7347170 & -0.340790 \\
\hline $\mathrm{H}$ & -0.5152870 & 1.9478530 & 561250 & $\mathrm{H}$ & -1.0513330 & -3.1522700 & -0 \\
\hline $\mathrm{C}$ & -4.4015420 & -1.94 & 0.26 & $\mathrm{C}$ & & 2.47 & 00 \\
\hline $\mathrm{H}$ & -4.9830630 & -2.6207360 & -0.3803480 & $\mathrm{H}$ & -1.3942710 & 2.6907980 & 2.9616910 \\
\hline $\mathrm{H}$ & -4.0579970 & -2.5228830 & 1.1271090 & $\mathrm{H}$ & -2.5455180 & 3.2753190 & 1.7345130 \\
\hline $\mathrm{H}$ & -5.07 & -1.15 & 40 & H & -3.1 & 2.475 & 3780 \\
\hline $\mathrm{C}$ & -3.7121920 & -0.6141930 & 950 & $\mathrm{C}$ & -3.1106310 & -3.682 & 2510 \\
\hline $\mathrm{H}$ & -2.8844110 & -0.1577780 & -2.3362560 & $\mathrm{H}$ & -3.0185580 & -4.6724400 & -0.233914 \\
\hline $\mathrm{H}$ & -4.2134840 & -1.3206190 & -2.4536720 & $\mathrm{H}$ & -3.0115670 & -3.80 & 3470 \\
\hline $\mathrm{H}$ & -4.43 & & & I & & & 970 \\
\hline $\mathrm{C}$ & 1.2124770 & 2.4515850 & 700 & $\mathrm{C}$ & -2.1320810 & -2.6382330 & -1.8 \\
\hline $\mathrm{O}$ & 2.2472550 & 1.8783770 & -0.9853020 & $\mathrm{H}$ & -1.3800680 & -1.9576260 & -2.279993 \\
\hline $\mathrm{O}$ & 0.0349470 & 2.048 & 65790 & $\mathrm{H}$ & 9770 & -3.6275 & -2.3 \\
\hline$r$ & 1.3989380 & 3.6651140 & -2.3297380 & $\mathrm{H}$ & -3.1202750 & -2.2976760 & -2.19856 \\
\hline $\mathrm{H}$ & 1.6731860 & 4.5140170 & -1.69 & $\mathrm{C}$ & -0.3587850 & 2.0163900 & -1.919859 \\
\hline $\mathrm{H}$ & 2.2181710 & 3.4980200 & -3.0333930 & $\mathrm{O}$ & -0.5163310 & 2.4954390 & -0.7454420 \\
\hline $\mathrm{H}$ & 0.4808850 & 3.9060320 & -2.8672750 & $\mathrm{O}$ & -0.6391220 & 0.7826510 & -2.089412 \\
\hline $\mathrm{C}$ & 2.8700690 & -2.4881190 & & $\mathrm{C}$ & & & \\
\hline $\mathrm{C}$ & 3.7007040 & -2.3945120 & 0.8192950 & $\mathrm{H}$ & 1.2721570 & 2.8384970 & -2.9978120 \\
\hline $\mathrm{C}$ & 3.6031310 & -1.2859080 & 1.6679080 & $\mathrm{H}$ & -0.1415470 & 2.4612180 & -4.0039100 \\
\hline$C_{1}$ & 2.6723950 & 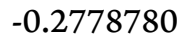 & & & 390 & 3.8940970 & -2.92701 \\
\hline $\mathrm{C}$ & 1.8060990 & -0.3571410 & 0.2971210 & $\mathrm{C}$ & 4.1523880 & -1.4824370 & -0.30857 \\
\hline $\mathrm{C}$ & 1.9336190 & -1.4834950 & -0.5507580 & $\mathrm{C}$ & 5.5163040 & -1.4223760 & -0.0243980 \\
\hline $\mathrm{H}$ & 2.9332600 & -3.3391560 & -0.9716680 & $C$ & 6.0334980 & -0.3439210 & 0.6991610 \\
\hline 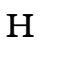 & 4.4251270 & -3.1770940 & 1.0267510 & $C_{0}+2$ & 5.1811640 & 0.6744000 & 1.1353090 \\
\hline $\mathrm{H}$ & 4.2600870 & -1.2044690 & 2.5300510 & $\mathrm{C}$ & 3.8169250 & 0.6163390 & 0.8492000 \\
\hline $\mathrm{H}$ & 2.6362420 & 0.5930290 & 2.0550540 & $\mathrm{C}$ & 3.2907630 & -0.4638700 & 0.1243120 \\
\hline $\mathrm{C}$ & 1.0027170 & -1.5659200 & -1.7186200 & $\mathrm{H}$ & 3.7424360 & -2.3180450 & -0.8663030 \\
\hline $\mathrm{O}$ & 0.0006670 & -0.7266960 & -1.6817360 & $\mathrm{H}$ & 6.1753670 & -2.2162390 & -0.3649410 \\
\hline $\mathrm{O}$ & 1.1728450 & -2.3673970 & -2.6506180 & $\mathrm{H}$ & 7.0957500 & -0.2979980 & 0.9235100 \\
\hline $\mathrm{H}$ & 1.8139500 & 0.8425590 & -0.3098360 & $\mathrm{H}$ & 5.5791590 & 1.5132860 & 1.6996280 \\
\hline
\end{tabular}




\begin{tabular}{|c|c|c|c|c|c|c|c|}
\hline $\mathrm{C}$ & 1.8170960 & -0.5650080 & -0.1843120 & $\mathrm{C}$ & 3.1106920 & -1.4926680 & -0.8170790 \\
\hline $\mathrm{O}$ & 1.1061740 & 0.4456170 & 0.2100130 & $\mathrm{H}$ & 2.4419580 & -2.2652820 & -1.2167540 \\
\hline O & 1.3702460 & -1.5686180 & -0.7714760 & $\mathrm{C}$ & 3.6226190 & -1.9640250 & 0.5494250 \\
\hline$H$ & 3.1552390 & 1.4055770 & 1.1900570 & $\mathrm{H}$ & 2.8082810 & -2.0720910 & 1.2721600 \\
\hline & & & & $\mathrm{H}$ & 4.1060700 & -2.9403130 & 0.4403390 \\
\hline & & & & $\mathrm{H}$ & 4.3669880 & -1.2774760 & 0.9674580 \\
\hline $\mathrm{C}$ & 1.7215190 & 1.9867490 & 0.1716260 & $\mathrm{C}$ & 4.2774530 & -1.3232820 & -1.8138900 \\
\hline 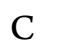 & 2.5005590 & 0.7960280 & 0.1981620 & $\mathrm{H}$ & 4.8265780 & -2.2658610 & -1.9100480 \\
\hline $\mathrm{C}$ & 2.3077670 & -0.2037600 & -0.7796620 & $\mathrm{H}$ & 3.9212630 & -1.0357620 & -2.8084760 \\
\hline $\mathrm{C}$ & 1.3088150 & 0.0214540 & -1.7746850 & $\mathrm{H}$ & 4.9792080 & -0.5555360 & -1.4681750 \\
\hline $\mathrm{C}$ & 0.6052840 & 1.2554240 & -1.8379020 & \multirow{2}{*}{\multicolumn{2}{|c|}{ Yts 1}} & & \\
\hline $\mathrm{C}$ & 0.8157070 & 2.2833360 & -0.8853510 & & & & \\
\hline $\mathrm{H}$ & 1.8483750 & 2.7120030 & 0.9697000 & $\mathrm{C}$ & 2.0459630 & 1.8733200 & 0.1640740 \\
\hline $\mathrm{H}$ & 3.2117040 & 0.6510740 & 1.0021320 & $\mathrm{C}$ & 2.5373980 & 0.5691870 & 0.3974020 \\
\hline 11 & 1.1108120 & -0.7445030 & -2.5170850 & $\mathrm{C}$ & 2.3095640 & -0.4899650 & -0.5479490 \\
\hline $\mathrm{H}$ & -0.1316960 & 1.4011210 & -2.6207120 & $\mathrm{C}$ & 1.5458990 & -0.2015740 & -1.6972210 \\
\hline $\mathrm{Ru}$ & 0.1648630 & 0.3233030 & 0.1837400 & $\mathrm{C}$ & 0.9561330 & 1.0842370 & -1.8606540 \\
\hline $\mathrm{C}$ & -0.1066080 & 0.7346330 & 2.3409290 & $\mathrm{C}$ & 1.2360210 & 2.1641320 & -0.9709950 \\
\hline $\mathrm{C}$ & -1.0955770 & 1.25 & 1.78 & 11 & 2370 & 160 & 320 \\
\hline $\mathrm{C}$ & -1.7182120 & -0.1595550 & -0.5117920 & $\mathrm{H}$ & 3.1014820 & 0.3758770 & 1.3017610 \\
\hline $\mathrm{C}$ & -2.5950310 & 0.6196450 & -1.2877260 & $\mathrm{H}$ & 1.3299010 & -0.9874350 & -2.4135740 \\
\hline $\mathrm{C}$ & -2.1328780 & -1.4685420 & -0.1915720 & $\mathrm{H}$ & 0.3013100 & 1.2609790 & -2.7075580 \\
\hline $\mathrm{C}$ & -3.8203830 & 0.1041940 & -1.7238480 & $\mathrm{Ru}$ & 0.2764520 & 0.4532210 & 0.1661670 \\
\hline $\mathrm{H}$ & -2.3339590 & 1.6408920 & -1.5538920 & $\mathrm{C}$ & -0.7990830 & 1.4589210 & 1.6020550 \\
\hline $\mathrm{C}$ & -3.3626400 & -1.9870650 & -0.6194100 & $\mathrm{C}$ & -1.7824370 & 1.2875170 & 0.7832530 \\
\hline $\mathrm{C}$ & -4.2123140 & -1.1994990 & -1.3919640 & $\mathrm{C}$ & -1.6950960 & -0.2150560 & -0.4452500 \\
\hline $\mathrm{H}$ & -4.4775000 & 0.7272180 & -2.3270510 & $\mathrm{C}$ & -2.3286680 & 0.0870950 & -1.6706650 \\
\hline $\mathrm{H}$ & -3.6349520 & -3.0027970 & -0.3424550 & $\mathrm{C}$ & -2.0003230 & -1.4624940 & 0.1523770 \\
\hline $\mathrm{H}$ & -5.1672550 & -1.5887850 & -1.7340660 & $\mathrm{C}$ & -3.2230320 & -0.8071800 & -2.2572640 \\
\hline $\mathrm{C}$ & -1.1831590 & -2.2657750 & 0.6227320 & $\mathrm{H}$ & -2.1297460 & 1.0346500 & -2.1630370 \\
\hline $\mathrm{O}$ & -1.4037530 & -3.4298060 & 1.0017680 & $\mathrm{C}$ & -2.9271410 & -2.3393570 & -0.4193930 \\
\hline $\mathrm{O}$ & -0.0704550 & -1.6363570 & 0.9117700 & $\mathrm{C}$ & -3.5424080 & -2.0182330 & -1.6282940 \\
\hline $\mathrm{C}$ & -2.3559750 & 2.0234650 & 1.7349240 & $\mathrm{H}$ & -3.6847070 & -0.5529940 & -3.2082770 \\
\hline $\mathrm{H}$ & -2.3179770 & 2.8174730 & 0.9822250 & $\mathrm{H}$ & -3.1329970 & -3.2818940 & 0.0811940 \\
\hline $\mathrm{H}$ & -3.2094810 & 1.3827780 & 1.4954470 & $\mathrm{H}$ & -4.2542260 & -2.7013740 & -2.0825020 \\
\hline $\mathrm{H}$ & -2.5389510 & 2.4864200 & 2.7118000 & $\mathrm{C}$ & -1.2199080 & -1.8487260 & 1.3584710 \\
\hline $\mathrm{C}$ & 0.7993180 & 0.3151660 & 3.4245490 & $\mathrm{O}$ & -1.5574900 & -2.7821690 & 2.1095690 \\
\hline $\mathrm{H}$ & 1.7623490 & 0.8333240 & 3.3615860 & $\mathrm{O}$ & -0.1384580 & -1.1448950 & 1.5444960 \\
\hline $\mathrm{H}$ & 0.3512870 & 0.5442730 & 4.3992770 & $\mathrm{C}$ & -3.0678000 & 1.9766410 & 0.4525540 \\
\hline $\mathrm{H}$ & 0.9936450 & -0.7613860 & 3.3842650 & $\mathrm{H}$ & -2.9865080 & 2.5584900 & -0.4717490 \\
\hline $\mathrm{C}$ & 0.1328190 & 3.6190470 & -0.9894860 & $\mathrm{H}$ & -3.9010940 & 1.2775930 & 0.3356050 \\
\hline $\mathrm{H}$ & -0.0164120 & 4.0686930 & -0.0042270 & $\mathrm{H}$ & -3.2990440 & 2.6735680 & 1.2666810 \\
\hline $\mathrm{H}$ & 0.7540330 & 4.3073250 & -1.5763310 & $\mathrm{C}$ & -0.4517800 & 2.1499110 & 2.8679020 \\
\hline $\mathrm{H}$ & -0.8333360 & 3.5349080 & -1.4939400 & $\mathrm{H}$ & 0.2949540 & 2.9332160 & 2.6944210 \\
\hline
\end{tabular}




\begin{tabular}{|c|c|c|c|c|c|c|c|}
\hline H & 1.3341100 & .6115540 & 70 & $\mathrm{C}$ & 2.88 & $-2.8 c$ & 1.4 \\
\hline $\mathrm{H}$ & -0.0168080 & 1.4484190 & 3.5900640 & $\mathrm{C}$ & 3.4541280 & -1.7363250 & 2.0510700 \\
\hline c & 0.7115970 & .5502130 & .2275430 & $\mathrm{C}$ & 3.2960000 & -0.4831350 & 1.4599980 \\
\hline$U$ & 0.5214500 & 310 & 925000 & C & 550 & 0 & 2 \\
\hline $\mathrm{H}$ & 1.4482730 & 90 & 150 & 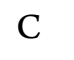 & 1.9697500 & -1.45 & -0.309915 \\
\hline$\theta$ & -0.2126380 & 30 & 70 & $\mathrm{H}$ & 540 & 240 & -0.2 \\
\hline $\mathrm{C}$ & 2.9129820 & 1.8761960 & 0.3713760 & $\mathrm{H}$ & 3.0088870 & -3.8470440 & 960 \\
\hline & 2.2029330 & -2.5868510 & 40 & $\mathrm{H}$ & 4.0278750 & & 530 \\
\hline C & 3.1547400 & -2.2 & 1.08 & $\mathrm{H}$ & 3.76 & 0.38 & 8520 \\
\hline $\mathrm{H}$ & 2.2460050 & -2.2019970 & 1.6898920 & $\mathrm{C}$ & 2.4306750 & 1.0526940 & -0.335263 \\
\hline 1 & 3.4873000 & -3.3295800 & 90 & $\mathrm{C}$ & 500 & 50 & 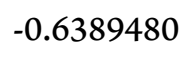 \\
\hline $\mathrm{H}$ & 3.9379730 & -1.68 & 570 & $\mathrm{C}$ & 1.0193760 & 2.8747050 & -1 \\
\hline C & 4.2228540 & -1.9582780 & -1.1839080 & $\mathrm{H}$ & 0.0856220 & 2.8282480 & -1.993738 \\
\hline $\mathrm{H}$ & 4.6484690 & -2.9649400 & -1.1100980 & $\mathrm{H}$ & 1.8236910 & 3.0809380 & -2.125440 \\
\hline & 4.05 & 30 & -2 & $\mathrm{H}$ & 00 & 3.742 & -0.7 \\
\hline $\mathrm{U}$ & $4.96 c$ & 10 & -0.8 & $\mathrm{C}$ & 80 & 00 & -0 \\
\hline & & & & $\mathrm{H}$ & 4.4027000 & 1.1329360 & -1.24903 \\
\hline & & & & $\mathrm{H}$ & 4.32 & 1.9 & 0.3 \\
\hline $\mathrm{Ru}$ & -0.6 & 0 & -0 . & $\mathrm{H}$ & & & \\
\hline C & -0.6873180 & 0.7094910 & 1.6863050 & $\mathrm{C}$ & 1.2124600 & -1.4339140 & -1.61895 \\
\hline C & -1.2708290 & 1.9652380 & 1.3255690 & $\mathrm{O}$ & 0.1791700 & -0.6589620 & -1.755368 \\
\hline $\mathrm{C}$ & -2.3259440 & 1.9146490 & 0.3806630 & 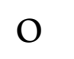 & 1.5676680 & -2.2101850 & -2.5239 \\
\hline $\mathrm{C}$ & -2.9731770 & 0.68 & -0 & \multirow{2}{*}{\multicolumn{2}{|c|}{$\mathbf{Y}$}} & & \\
\hline $\mathrm{C}$ & -2.4595900 & -0.5431380 & 0.4019440 & & & & \\
\hline $\mathrm{C}$ & -1.2215030 & -0.5057600 & 1.1560140 & $\mathrm{C}$ & 50 & 0.3 & 1. \\
\hline $\mathrm{H}$ & 0.1854680 & 0.677 & 0 & $\mathrm{C}$ & 60 & 0 & 5030 \\
\hline $\mathrm{H}$ & -2.7059490 & 2.8469280 & -0.026 & C & 1.12 & 2.18 & 0.5 \\
\hline $\mathrm{H}$ & -3.8198950 & 0.7371750 & -0.6808570 & $\mathrm{C}$ & -0.1957790 & 1.6942880 & 0.9244450 \\
\hline $\mathrm{H}$ & -0.7293620 & -1.4414740 & 1.4015660 & $C$ & -0.4126000 & 0.6632120 & 1.8767330 \\
\hline $\mathrm{C}$ & -0.7442380 & 3.26 & & $\mathrm{C}$ & & & \\
\hline $\mathrm{H}$ & 0.3317560 & 3.2151610 & 2.053 & $\mathrm{H}$ & 2.8493770 & -0.23 & 2.2954070 \\
\hline $\mathrm{H}$ & -1.2365130 & 3.5010990 & 2.8166420 & $\mathrm{H}$ & 3.2132480 & 1.6870060 & 0.7829330 \\
\hline-7 & -0.9433690 & 4.0920560 & .1762110 & $\Pi$ & -1.0594920 & 2.1803180 & 0.4829810 \\
\hline $\mathrm{C}$ & -3.0508090 & -1.8903330 & .028 & 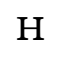 & -1.4229250 & 0.3946 & 2.161 \\
\hline $\mathrm{H}$ & -2.2059910 & -2.5372560 & -0.2452650 & $\mathrm{Ru}$ & 0.6227860 & -0.1647660 & 0.1539510 \\
\hline $\mathrm{C}$ & -3.7261830 & -2.5162620 & 1.2664310 & $\mathrm{O}$ & 1.9976380 & -1.2179200 & -1.327779 \\
\hline $\mathrm{H}$ & -4.0840650 & -3.5246820 & 1.0327420 & $\mathrm{C}$ & 3.0681560 & -1.7989070 & -1.11949 \\
\hline 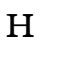 & -4.5859230 & -1.9169420 & 1.5879270 & $\mathrm{O}$ & 3.5738260 & -1.9309240 & 0.0996820 \\
\hline $\mathrm{H}$ & -3.0324000 & -2.5937370 & 2.1101860 & $\mathrm{C}$ & 3.8938450 & -2.4125030 & -2.2026340 \\
\hline 4 & -4.0172910 & -1.8453920 & -1.1603240 & $\mathrm{H}$ & 3.9592680 & -3.4928270 & -2.035271 \\
\hline $\mathrm{H}$ & -4.3368900 & -2.8607840 & -1.4160720 & $\mathrm{H}$ & 3.4450810 & -2.2136680 & -3.175360 \\
\hline $\mathrm{H}$ & -3.5486520 & -1.4086610 & -2.0488370 & $\mathrm{H}$ & 4.9103170 & -2.0090050 & -2.1639590 \\
\hline $\mathrm{H}$ & -4.9190330 & -1.2666210 & -0.9298300 & $\mathrm{C}$ & -0.4956310 & -1.8996750 & 0.1662910 \\
\hline $\mathrm{C}$ & 2.1642440 & -2.7177740 & 0.2709660 & $\mathrm{C}$ & -1.8391530 & -1.9682730 & -0.001865 \\
\hline
\end{tabular}




\begin{tabular}{|c|c|c|c|c|c|c|c|}
\hline $\mathrm{C}$ & -2.7333010 & -0.7738420 & -0.0964450 & $\mathrm{H}$ & 6010 & 2.6310880 & 480 \\
\hline C & -3.8567990 & -0.7200140 & 0.7565800 & $\mathrm{H}$ & 2.9944230 & 0.4174090 & 1.6157140 \\
\hline $\mathrm{C}$ & -2.5783840 & 0.2787110 & -1.0279810 & $\mathrm{H}$ & 2.1952040 & -0.7167200 & -2.4955840 \\
\hline C & -4.7623880 & 0.3381310 & 0.7287820 & $\mathrm{H}$ & 1.1479310 & 1.4850520 & -2.9228650 \\
\hline $\mathrm{H}$ & -4.0129070 & -1.5277610 & 1.4666740 & $\mathrm{Ru}$ & 0.5135340 & 0.3235210 & -0.0365990 \\
\hline C & -3.5178330 & 1.3231850 & -1.0762230 & $\mathrm{C}$ & -0.4938170 & 0.5316400 & 1.5633680 \\
\hline $\mathrm{C}$ & -4.5931220 & 1.3743900 & -0.1936040 & $\mathrm{C}$ & -1.6583690 & 0.9462340 & 0.8879210 \\
\hline $\mathrm{H}$ & -5.6014670 & 0.3490920 & 1.4197330 & $\mathrm{C}$ & -2.6248700 & 0.1394460 & 0.0964790 \\
\hline $\mathrm{H}$ & -3.3914960 & 2.1004470 & -1.8242710 & $\mathrm{C}$ & -3.8023190 & 0.7771850 & -0.3659710 \\
\hline $\mathrm{H}$ & -5.2975080 & 2.2004910 & -0.2350550 & $\mathrm{C}$ & -2.5631390 & -1.2724920 & -0.0909730 \\
\hline C & -1.4877180 & 0.3261920 & -2.0674770 & $\mathrm{C}$ & -4.8653180 & 0.0738620 & -0.9277330 \\
\hline $\mathrm{O}$ & -1.7988230 & 0.6078260 & -3.2422560 & $\mathrm{H}$ & -3.9040960 & 1.8503600 & -0.2741710 \\
\hline $\mathrm{O}$ & -0.2553180 & 0.1123430 & -1.7332820 & $\mathrm{C}$ & -3.6606740 & -1.9711220 & -0.6117150 \\
\hline $\mathrm{C}$ & -2.5808240 & -3.3085860 & -0.0247470 & $\mathrm{C}$ & -4.8147270 & -1.3157020 & -1.0342630 \\
\hline $\mathrm{H}$ & -2.7263220 & -3.7353580 & 0.9776850 & $\mathrm{H}$ & -5.7420450 & 0.6200720 & -1.2655960 \\
\hline $\mathrm{H}$ & -3.5727310 & -3.2001710 & -0.4735810 & $\mathrm{H}$ & -3.5790490 & -3.0497350 & -0.7078760 \\
\hline $\mathrm{H}$ & -2.0407700 & -4.0569110 & -0.6134710 & $\mathrm{H}$ & -5.6476800 & -1.8763800 & -1.4479230 \\
\hline $\mathrm{C}$ & 0.3024990 & -3.1840450 & 0.3368850 & $\mathrm{C}$ & -1.3278850 & -2.0956210 & 0.0959060 \\
\hline $\mathrm{H}$ & -0.2664370 & -3.9673390 & 0.8517690 & $\mathrm{O}$ & -1.3822050 & -3.2251680 & 0.6123630 \\
\hline $\mathrm{H}$ & 0.6065450 & -3.5964280 & -0.6358940 & $\mathrm{O}$ & -0.2390590 & -1.6201400 & 67610 \\
\hline $\mathrm{H}$ & 1.2189600 & -3.0262430 & 0.9105630 & $\mathrm{C}$ & -2.1138200 & 2.3622950 & 1.2441470 \\
\hline $\mathrm{C}$ & 0.5236820 & -1.1401890 & 3.4379130 & $\mathrm{H}$ & -2.2632980 & 2.9649140 & 0.3421720 \\
\hline $\mathrm{H}$ & 1.3757580 & -1.8252870 & 3.4483410 & $\mathrm{H}$ & -3.0593290 & 2.3600480 & 1.8005060 \\
\hline $\mathrm{H}$ & 0.4413470 & -0.6928840 & 4.4364880 & $\mathrm{H}$ & -1.3655150 & 2.8773640 & 1.8488090 \\
\hline $\mathrm{H}$ & -0.3890130 & -1.7139260 & 3.2555580 & $\mathrm{C}$ & -0.1253820 & 0.8057390 & 2.9824710 \\
\hline $\mathrm{C}$ & 1.2481900 & 3.4057610 & -0.3088690 & $\mathrm{H}$ & 0.9069200 & 1.1510960 & 3.0957440 \\
\hline $\mathrm{H}$ & 0.4500840 & 3.3234760 & -1.0592630 & $\mathrm{H}$ & -0.8051910 & 1.5050350 & 3.4823700 \\
\hline $\mathrm{C}$ & 2.5861640 & 3.5062120 & -1.0498590 & $\mathrm{H}$ & -0.1977610 & -0.1497720 & 3.5237470 \\
\hline $\mathrm{H}$ & 2.7915050 & 2.6059640 & -1.6389960 & $\mathrm{C}$ & 0.7373900 & 3.5280600 & -1.1198050 \\
\hline $\mathrm{H}$ & 2.5656900 & 4.3590600 & -1.7361820 & $\mathrm{H}$ & 0.3695950 & 3.9690410 & -0.1900890 \\
\hline $\mathrm{H}$ & 3.4227090 & 3.6623870 & -0.3593240 & $\mathrm{H}$ & 1.4553560 & 4.2299440 & -1.5629790 \\
\hline $\mathrm{C}$ & 0.9770640 & 4.6817470 & 0.5152930 & $\mathrm{H}$ & -0.0980360 & 3.4239430 & -1.8180220 \\
\hline $\mathrm{H}$ & 0.9808980 & 5.5629850 & -0.1353690 & $\mathrm{C}$ & 3.2977880 & -1.7118090 & -0.2147370 \\
\hline $\mathrm{H}$ & 0.0051620 & 4.6389810 & 1.0183110 & $\mathrm{H}$ & 2.7467340 & -2.4053950 & -0.8625420 \\
\hline $\mathrm{H}$ & 1.7490860 & 4.8213860 & 1.2812200 & $\mathrm{C}$ & 3.2195600 & -2.2481070 & 1.2205080 \\
\hline $\mathrm{H}$ & 2.9446580 & -1.5110910 & 0.7247550 & $\mathrm{H}$ & 2.1895920 & -2.2581660 & 1.5913230 \\
\hline \multirow{2}{*}{\multicolumn{2}{|c|}{ Yts2 }} & & & $\mathrm{H}$ & 3.5984140 & -3.2749590 & 1.2516230 \\
\hline & & & & $\mathrm{H}$ & 3.8290670 & -1.6562470 & 1.9126130 \\
\hline $\mathrm{C}$ & 2.0103240 & 1.9091120 & 0.3845490 & $\mathrm{C}$ & 4.7575720 & -1.6728630 & -0.7105680 \\
\hline $\mathrm{C}$ & 2.5960170 & 0.6363980 & 0.6320120 & $\mathrm{H}$ & 5.2073700 & -2.6697460 & -0.6462840 \\
\hline $\mathrm{C}$ & 2.6560870 & -0.3453290 & -0.4051030 & $\mathrm{H}$ & 4.8206530 & -1.3414690 & -1.7525610 \\
\hline $\mathrm{C}$ & 2.1794950 & 0.0287330 & -1.7053130 & $\mathrm{H}$ & 5.3613990 & -0.9904240 & -0.1007070 \\
\hline $\mathrm{C}$ & 1.5670380 & 1.2618660 & -1.9476460 & \multirow{2}{*}{\multicolumn{2}{|c|}{ Y3 }} & & \\
\hline C & 1.4091030 & 2.2043410 & -0.8740400 & & & & \\
\hline
\end{tabular}




\begin{tabular}{|c|c|c|c|c|c|c|c|}
\hline C & 2.1502180 & 1.7417880 & 420 & $\mathrm{C}$ & 4.67 & 850 & -0. \\
\hline $\mathrm{C}$ & 2.6821590 & 0.4320150 & 0.7673310 & $\mathrm{H}$ & 5.0406270 & -2.9581270 & -0.8040000 \\
\hline$C$ & 2.6998820 & -0.4541310 & -0.3326450 & $\mathrm{H}$ & 4.7303350 & -1.5374600 & -1.8139480 \\
\hline & 2.2751670 & 0.0442520 & -1.6142910 & $\mathrm{H}$ & 5.3507130 & -1.3326140 & 0.1659240 \\
\hline & 1.7680810 & 1.3285840 & -1.7827050 & \multirow{2}{*}{\multicolumn{2}{|c|}{ Yts3 }} & & \\
\hline C & 1.6412670 & 2.1975500 & -0.6431910 & & & & \\
\hline$\theta$ & 2.1110060 & 2.4067380 & 1.4660800 & C & 1.7697670 & 1.8411940 & 0.9931090 \\
\hline & 3.0512270 & 0.1210020 & 1.7372920 & $\mathrm{C}$ & 2.4740730 & 0.636 & 0.70 \\
\hline H & 2.2819800 & -0.6380030 & -2.4596080 & $\mathrm{C}$ & 2.3833490 & 0.0438170 & -0.5913160 \\
\hline $\mathrm{H}$ & 1.4393660 & 1.6693840 & -2.7587380 & $\mathrm{C}$ & 1.6998450 & 0.7668260 & -1.6347070 \\
\hline $\mathrm{Ru}$ & 0.4808510 & 0.3624740 & 0.0290360 & $\mathrm{C}$ & 1.0351760 & 1.9626790 & -1.3616760 \\
\hline C & -0.6329580 & 0.7137200 & 1.463 & $\mathrm{C}$ & 0.985 & 2.479 & 5930 \\
\hline $\mathrm{C}$ & -1.5750500 & 1.0800470 & 0.4453050 & $\mathrm{H}$ & 1.7745090 & 2.2480900 & 1.9988810 \\
\hline C & -2.6566960 & 0.1804070 & -0.0596850 & $\mathrm{H}$ & 3.0097880 & 0.1314860 & 1.5014220 \\
\hline C & -3.8788690 & 0.7663220 & -0.4650780 & $\mathrm{H}$ & 1.6318340 & 0.3241000 & -2.6232600 \\
\hline $\mathrm{C}$ & -2.5974680 & -1.2424480 & -0.0916730 & $\mathrm{H}$ & 0.4432590 & 2.4432640 & 3470 \\
\hline $\mathrm{C}$ & -4.9890780 & 0.0087320 & -0.8309870 & $\mathrm{Ru}$ & 0.3747000 & 0.3762090 & 0.2484150 \\
\hline $\mathrm{H}$ & -3.9783850 & 1.8436690 & -0.4804590 & $\mathrm{C}$ & -0.2418590 & -0.5683970 & 1.9123680 \\
\hline $\mathrm{C}$ & -3.7394940 & -1.9909640 & -0.4208670 & $\mathrm{C}$ & -1.5129700 & -0.9082350 & 1.5934810 \\
\hline C & -4.9348670 & -1.3843830 & -0.7916500 & $\mathrm{C}$ & -2.1075980 & -0.1464630 & 0.4252840 \\
\hline $\mathrm{H}$ & -5.9015700 & 0.5160630 & -1.1332760 & $\mathrm{C}$ & -2.5730120 & 1.1674040 & 0.6533380 \\
\hline $\mathrm{H}$ & -3.6560060 & -3.0728370 & -0.4121820 & $\mathrm{C}$ & -2.3659260 & -0.7458380 & -0.8406970 \\
\hline $\mathrm{H}$ & -5.7985520 & -1.9858960 & -1.0595620 & $\mathrm{C}$ & -3.2879880 & 1.8648310 & -0.3275200 \\
\hline C & -1.3462010 & -2.0525550 & 0.0572790 & $\mathrm{H}$ & -2.4133930 & 1.6173290 & 1.6290250 \\
\hline $\mathrm{O}$ & -1.3923070 & -3.2107330 & 0.5089550 & $\mathrm{C}$ & -3.1069020 & -0.0495480 & -1.7972290 \\
\hline o & -0.2455840 & -1.5522930 & -0.4283860 & $\mathrm{C}$ & -3.5647300 & 1.2491130 & -1.5467380 \\
\hline 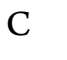 & -1.8915260 & 2.5770840 & 0.4311630 & $\mathrm{H}$ & -3.6399170 & 2.8723690 & -0.1266620 \\
\hline $\mathrm{H}$ & -2.0747290 & 2.9465510 & -0.5836570 & $\mathrm{H}$ & -3.3106540 & -0.5243890 & -2.7529730 \\
\hline $\mathrm{H}$ & -2.7769320 & 2.8118080 & 1.0366240 & $\mathrm{H}$ & -4.1326980 & 1.7766980 & -2.3081980 \\
\hline $\mathrm{H}$ & -1.0561900 & 3.1469310 & 0.8418790 & $\mathrm{C}$ & -1.7628370 & -2.0903890 & -1.2099040 \\
\hline C & -0.5983400 & 0.9834420 & 2.9175780 & $\mathrm{O}$ & -2.5283530 & -2.9719000 & -1.6679530 \\
\hline $\mathrm{H}$ & 0.3972110 & 0.8618450 & 3.3528510 & $\mathrm{O}$ & -0.5026440 & -2.1923380 & -1.0527690 \\
\hline $\mathrm{H}$ & -0.9829340 & 1.9874610 & 3.1434180 & $\mathrm{C}$ & -2.4221240 & -1.9012920 & 2.2738970 \\
\hline $\mathrm{H}$ & -1.2796520 & 0.2744270 & 3.4121420 & $\mathrm{H}$ & -3.2945550 & -1.3990380 & 2.7132870 \\
\hline C & 1.1827610 & 3.6188880 & -0.8289230 & $\mathrm{H}$ & -2.8109110 & -2.6181000 & 1.5386740 \\
\hline $\mathrm{H}$ & 0.9163160 & 4.0843370 & 0.1225980 & $\mathrm{H}$ & -1.9109470 & -2.4606240 & 3.0612610 \\
\hline $\mathrm{H}$ & 1.9963820 & 4.2069820 & -1.2722060 & $\mathrm{C}$ & 0.5349010 & -0.9952930 & 3.1234000 \\
\hline $\mathrm{H}$ & 0.3259740 & 3.6798850 & -1.5053710 & $\mathrm{H}$ & 0.9462620 & -0.1238270 & 3.6477040 \\
\hline $\mathrm{C}$ & 3.2328450 & -1.8764720 & -0.2420400 & $\mathrm{H}$ & -0.0752590 & -1.5529050 & 3.8445730 \\
\hline $\mathrm{H}$ & 2.6052390 & -2.4871360 & -0.9039670 & $\mathrm{H}$ & 1.3880330 & -1.6269110 & 2.8484460 \\
\hline C & 3.1612800 & -2.4847330 & 1.1638780 & $\mathrm{C}$ & 0.2048410 & 3.7258050 & 0.2851140 \\
\hline $\mathrm{H}$ & 2.1460540 & -2.4401000 & 1.5713370 & $\mathrm{H}$ & -0.0863170 & 3.7646530 & 1.3380500 \\
\hline 5 & 3.4622300 & -3.5368290 & 1.1262640 & $\mathrm{H}$ & 0.8231560 & 4.6080530 & 0.0747490 \\
\hline $\mathrm{H}$ & 3.8355420 & -1.9790890 & 1.8641640 & $\mathrm{H}$ & -0.6934080 & 3.7935040 & -0.3342670 \\
\hline
\end{tabular}




\begin{tabular}{|c|c|c|c|c|c|c|c|}
\hline $\mathrm{C}$ & 3.0867020 & -1.2570060 & -0.9467350 & $\mathrm{H}$ & 6240 & 8740 & 3.7138820 \\
\hline $\mathrm{H}$ & 2.4939010 & -1.7242870 & -1.7424680 & $\mathrm{H}$ & 0.3762630 & -2.7250070 & 2.4977640 \\
\hline $\mathrm{C}$ & 3.1967400 & -2.2591290 & 0.2086900 & $\mathrm{C}$ & -0.4563760 & 3.8523180 & 0.3478860 \\
\hline $\mathrm{H}$ & 2.2160430 & -2.4898970 & 0.6359750 & $\mathrm{H}$ & -0.9153140 & 3.7786830 & 1.3381230 \\
\hline $\mathrm{H}$ & 3.6312250 & -3.1948510 & -0.1584080 & $\mathrm{H}$ & 0.1304470 & 4.7790380 & 0.3244910 \\
\hline $\mathrm{H}$ & 3.8467950 & -1.8923030 & 1.0105920 & $\mathrm{H}$ & -1.2468660 & 3.9360250 & -0.4025020 \\
\hline $\mathrm{C}$ & 4.4768510 & -0.9268460 & -1.5292050 & $\mathrm{C}$ & 3.2433980 & -0.6425750 & -0.7624580 \\
\hline $\mathrm{H}$ & 4.9727060 & -1.8439470 & -1.8648880 & $\mathrm{H}$ & 2.8995430 & -1.1174690 & -1.6886050 \\
\hline 10 & 4.4036910 & -0.2497700 & -2.3869220 & $\mathrm{C}$ & 3.3952870 & -1.7377630 & 0.3007070 \\
\hline $\mathrm{H}$ & 5.1157600 & -0.4 & 050 & $\mathrm{H}$ & 2.4425490 & -2.2380040 & 0.4945200 \\
\hline & & & & $\mathrm{H}$ & 4.1066490 & -2.4926580 & -0.0509820 \\
\hline 4 & & & & $\mathrm{H}$ & 3.7824190 & -1.3440590 & 1.2470140 \\
\hline $\mathrm{C}$ & 1.1879890 & 2.0652290 & 1.1290790 & $\mathrm{C}$ & 4.5968570 & 0.0383690 & -1.0551390 \\
\hline $\mathrm{C}$ & 2.0860270 & 0.9867540 & 0.8876350 & $\mathrm{H}$ & 5.3328030 & -0.7070760 & -1.3749730 \\
\hline $\mathrm{C}$ & 2.2240920 & 0.4308250 & -0.4202190 & $\mathrm{H}$ & 4.5078400 & 0.7874530 & -1.8493400 \\
\hline $\mathrm{C}$ & 1.3948500 & 0.9831520 & -1.4362790 & 11 & 0 & 0 & 000 \\
\hline $\mathrm{C}$ & 0.5554540 & 2.1099540 & -1.2105960 & \multirow{2}{*}{\multicolumn{2}{|c|}{ Yts4 }} & & \\
\hline $\mathrm{C}$ & 0.4359630 & 2.6738090 & 0.0733460 & & & & \\
\hline $\mathrm{H}$ & 1.0797210 & 2.4443980 & 2.1405820 & $\mathrm{Ru}$ & 0.7461730 & -0.1241910 & 25330 \\
\hline $\mathrm{H}$ & 2.6524540 & 0.5806510 & 1.7175530 & $\mathrm{C}$ & -0.2631760 & 0.4176690 & 1.9588110 \\
\hline $\mathrm{H}$ & 1.4087970 & 0.5300730 & -2.4222440 & $\mathrm{C}$ & 0.9995520 & -0.1312500 & 2.3422930 \\
\hline $\mathrm{H}$ & -0.0562720 & 2.4871700 & -2.0234210 & $\mathrm{C}$ & 2.1457610 & 0.4694210 & 1.7552300 \\
\hline $\mathrm{Ru}$ & 0.0579760 & 0.3403950 & 0.3054900 & $\mathrm{C}$ & & 1.699 & 4990 \\
\hline $\mathrm{C}$ & -0.3393390 & -0.9359110 & 1.6739000 & $\mathrm{C}$ & 0.8664790 & 2.2550790 & 0.6501070 \\
\hline $\mathrm{C}$ & -1.6726240 & -0.5273090 & 1.5282080 & $\mathrm{C}$ & -0.3141790 & 1.5187150 & 1.0597190 \\
\hline $\mathrm{C}$ & -2.1314180 & -0.3921550 & 0.1451970 & $\mathrm{H}$ & -1.1837850 & -0.0415180 & 2.3014580 \\
\hline $\mathrm{C}$ & -3.2456600 & 0.4439040 & -0.1942650 & $\mathrm{H}$ & 3.1187660 & 0.0247870 & 1.9410850 \\
\hline $\mathrm{C}$ & -1.4026220 & -1.0607340 & -0.9111750 & $\mathrm{H}$ & 3.0216620 & 2.1273140 & 0.6480820 \\
\hline $\mathrm{C}$ & -3.5854560 & 0.6671530 & -1.5061390 & $\mathrm{H}$ & -1.2850250 & 1.8725860 & 0.7284640 \\
\hline $\mathrm{H}$ & -3.8184290 & 0.9095530 & 0.6006090 & $\mathrm{C}$ & 1.1072950 & -1.2849960 & 3.3016870 \\
\hline $\mathrm{C}$ & -1.7634330 & -0.7341360 & -2.2604420 & $\mathrm{H}$ & 0.2592690 & -1.9688690 & 3.2018530 \\
\hline $\mathrm{C}$ & -2.8195160 & 0.0942420 & -2.5548190 & $\mathrm{H}$ & 1.1144020 & -0.9152360 & 4.3353240 \\
\hline $\mathrm{H}$ & -4.4376500 & 1.2976110 & -1.7444510 & $\mathrm{H}$ & 2.0326650 & -1.8468570 & 3.1443330 \\
\hline-7 & -1.2150860 & -1.2286990 & -3.0557230 & $\mathrm{C}$ & 0.6918440 & 3.5280330 & -0.1589620 \\
\hline $\mathrm{H}$ & -3.0930660 & 0.2845710 & -3.5887240 & $\mathrm{H}$ & -0.1306570 & 3.3384280 & -0.8622620 \\
\hline $\mathrm{C}$ & -0.6706910 & -2.4184870 & -0.7678320 & $\mathrm{C}$ & 0.2477770 & 4.6767320 & 0.7708400 \\
\hline $\mathrm{O}$ & -1.1057520 & -3.2136460 & 0.1078120 & $\mathrm{H}$ & 0.0377100 & 5.5796500 & 0.1871080 \\
\hline $\mathrm{O}$ & 0.2518740 & -2.6455650 & -1.5975470 & $\mathrm{H}$ & 1.0342430 & 4.9162100 & 1.4962790 \\
\hline $\mathrm{C}$ & -2.5677920 & -0.1496270 & 2.6847940 & $\mathrm{H}$ & -0.6590580 & 4.4183360 & 1.3279240 \\
\hline $\mathrm{H}$ & -2.8664660 & 0.9025890 & 2.6374290 & $\mathrm{C}$ & 1.9280170 & 3.9272080 & -0.9720340 \\
\hline $\mathrm{H}$ & -3.4832250 & -0.7526580 & 2.6701080 & $\mathrm{H}$ & 1.7001500 & 4.8021850 & -1.5896270 \\
\hline $\mathrm{H}$ & -2.0640180 & -0.3112430 & 3.6402570 & $\mathrm{H}$ & 2.2506330 & 3.1199830 & -1.6385190 \\
\hline $\mathrm{C}$ & 0.3174140 & -1.6648650 & 2.7830230 & $\mathrm{H}$ & 2.7711400 & 4.1936160 & -0.3244530 \\
\hline $\mathrm{H}$ & 1.3362750 & -1.3160450 & 2.9691770 & $\mathrm{C}$ & -3.7781220 & 1.0088300 & -1.0730940 \\
\hline
\end{tabular}




\begin{tabular}{|c|c|c|c|c|c|c|c|}
\hline $\mathrm{C}$ & -4.96 & 0.8 & 1650 & $\mathrm{H}$ & 0.8 & -1 . & 90 \\
\hline $\mathrm{C}$ & -5.0850020 & -0.2665270 & 0.4892320 & $\mathrm{H}$ & 1.8415490 & -0.6668700 & 4.2458880 \\
\hline $\mathrm{C}$ & -4.0492110 & -1.1586030 & 0.6574750 & $\mathrm{H}$ & 2.6050600 & -1.6179040 & 2.9669660 \\
\hline $\mathrm{C}$ & -2.7839830 & -1.0066160 & .0102580 & $\mathrm{C}$ & 0.3890450 & 3.5662930 & 0.1961420 \\
\hline C & -2.6862800 & 0.1214570 & -0.9128 & $\mathrm{H}$ & -0.5135400 & 3.3057450 & -0.7651610 \\
\hline $\mathrm{H}$ & -3.6617680 & 1.8323520 & -1.7715470 & $\mathrm{C}$ & 0.0067980 & 4.6947460 & 0.7848720 \\
\hline $\mathrm{H}$ & -5.7831380 & 1.5418470 & -0.5088570 & $\mathrm{H}$ & -0.3552690 & 5.5706270 & 0.2353920 \\
\hline $\mathrm{H}$ & -6.0126670 & -0.4192520 & .0367890 & $\mathrm{H}$ & 0.8730000 & 5.003 & 460 \\
\hline $\mathrm{H}$ & -4.1860300 & -1.9961810 & 1.3330240 & $\mathrm{H}$ & -0.7837890 & 4.37 & 1980 \\
\hline $\mathrm{C}$ & -1.7658070 & -1.9798040 & 0.1209380 & $\mathrm{C}$ & 1.4541420 & 4.0425160 & -1.1902790 \\
\hline $\mathrm{C}$ & -0.4443170 & -1.7770240 & -0.3689580 & $\mathrm{H}$ & 1.0681630 & 4.8862200 & -1.7717020 \\
\hline $\mathrm{C}$ & 0.2876750 & -2.9591660 & -0.9770640 & $\mathrm{H}$ & 1.7347590 & 3.2510660 & -1.8935650 \\
\hline $\mathrm{H}$ & 0.9168800 & -2.6229010 & -1.8 & $\mathrm{H}$ & 2.36 & 40 & 7470 \\
\hline $\mathrm{H}$ & -0.4020910 & -3.7155460 & -1.3685070 & $\mathrm{C}$ & -4.0740390 & 0.6309160 & -1.1006870 \\
\hline $\mathrm{H}$ & 0.9440470 & -3.4396620 & -0.2425600 & $\mathrm{C}$ & -5.1967060 & 0.3867380 & -0.3502560 \\
\hline $\mathrm{C}$ & -2.0923260 & -3.3180660 & 0.7666470 & $\mathrm{C}$ & -5.1478580 & -0.63 & 1030 \\
\hline $\mathrm{H}$ & -2.9323900 & 840 & 0.2 & $\mathrm{C}$ & 33140 & 3070 & 1790 \\
\hline $\mathrm{H}$ & -2.3695640 & -3.2030100 & 1.8239740 & $\mathrm{C}$ & -2.7970630 & -1.1514760 & 0.1141230 \\
\hline $\mathrm{H}$ & -1.2384540 & -3.9985460 & 0.7494910 & $\mathrm{C}$ & -2.8705280 & -0.1046830 & 7040 \\
\hline $\mathrm{C}$ & -1.54 & 0.3 & -1 . & $\mathrm{H}$ & -4.0798160 & 1.3973240 & 080 \\
\hline $\mathrm{O}$ & -0.4762630 & -0.5147440 & -1.6349040 & $\mathrm{H}$ & -6.1090770 & 0.9539480 & -0.5079040 \\
\hline $\mathrm{O}$ & -1.4865720 & 1.1447800 & -2.7114460 & $\mathrm{H}$ & -6.0366130 & -0.8378270 & 1.2453740 \\
\hline $\mathrm{C}$ & 3.3268520 & -1.5184590 & -1.0536930 & $\mathrm{H}$ & -4.0259340 & -2.1440060 & 1.6320890 \\
\hline $\mathrm{O}$ & 2.3681880 & -0.7850080 & -1.3255400 & $\mathrm{C}$ & -1.6415800 & -1.8913370 & 0.3066050 \\
\hline $\mathrm{O}$ & 3.4144980 & -2.1692720 & 0.0994340 & $\mathrm{C}$ & -0.4595300 & -1.6531060 & -0.5177850 \\
\hline $\mathrm{C}$ & 4.4705410 & -1.7571870 & -1.9838460 & $\mathrm{C}$ & 0.1546080 & -2.8836660 & -1.1723750 \\
\hline $\mathrm{H}$ & 4.3341890 & -1.1870360 & -2.902 & $\mathrm{H}$ & 0. & -2 . & 060 \\
\hline 11 & 5.4050870 & -1.4656590 & -1.4937080 & $\mathrm{H}$ & -0.6177030 & -3.4488400 & -1.7132720 \\
\hline $\mathrm{H}$ & 4.5358270 & -2.8261670 & -2.2113030 & $\mathrm{H}$ & 0.5900800 & -3.5502030 & -0.4237390 \\
\hline $\mathrm{H}$ & 2.6211510 & -1.9498200 & 0.6357360 & $\mathrm{C}$ & -1.5221800 & -2.9845570 & 1.3436480 \\
\hline \multirow{2}{*}{\multicolumn{2}{|c|}{$\mathbf{Y}^{\prime}$}} & & & $\mathrm{H}$ & -1.8868010 & -3.9603360 & 0.988 \\
\hline & & & & $\mathrm{H}$ & -2.0819890 & -2.7517300 & 2.2556310 \\
\hline $\mathrm{Ru}$ & 0.8430960 & -0.0286460 & 0.0700800 & $\mathrm{H}$ & -0.4781070 & -3.1303650 & 1.6417420 \\
\hline $\mathrm{C}$ & 0.0008140 & 0.4172440 & 2.0385760 & $\mathrm{C}$ & -1.7519820 & 0.1948880 & -1.7133040 \\
\hline $\mathrm{C}$ & 1.3435710 & -0.0085340 & 2.2670070 & $\mathrm{O}$ & -0.6050900 & -0.6182200 & -1.5389590 \\
\hline $\mathrm{C}$ & 2.3469260 & 0.6813520 & 1.5265320 & $\mathrm{O}$ & -1.6181240 & 1.0731650 & -2.5798360 \\
\hline $\mathrm{C}$ & 2.0876810 & 1.8884520 & 0.7827550 & $\mathrm{C}$ & 3.2618860 & -1.5695860 & -1.1296480 \\
\hline $\mathrm{C}$ & 0.7773460 & 2.3241080 & 0.5865760 & $\mathrm{O}$ & 2.3748070 & -0.7393920 & -1.3751630 \\
\hline $\mathrm{C}$ & -0.2684490 & 1.5012580 & 1.1562630 & $\mathrm{O}$ & 3.2941360 & -2.2539140 & 0.0047890 \\
\hline $\mathrm{H}$ & -0.8234820 & -0.1156610 & 2.4996610 & $\mathrm{C}$ & 4.3688250 & -1.8892150 & -2.0788850 \\
\hline H & 3.3713980 & 0.3248650 & 1.5823670 & $\mathrm{H}$ & 4.2618870 & -1.3071400 & -2.9936140 \\
\hline $\mathrm{H}$ & 2.9213090 & 2.3895680 & 0.3040020 & $\mathrm{H}$ & 5.3305320 & -1.6688680 & -1.6042130 \\
\hline $\mathrm{H}$ & -1.3024980 & 1.7580410 & 0.9469110 & $\mathrm{H}$ & 4.3521050 & -2.9591280 & -2.3097510 \\
\hline$r$ & 1.6840730 & -1.0980720 & 3.2482310 & $\mathrm{H}$ & 2.5180800 & -1.9842340 & 0.5482890 \\
\hline
\end{tabular}




\section{Yts5'}

$\begin{array}{llll}\mathrm{Ru} & 0.9254970 & -0.3597350 & 0.3310610 \\ \mathrm{C} & 2.5611830 & -1.3855380 & 1.2791620 \\ \mathrm{C} & 2.2595330 & -2.2449850 & 0.1706670 \\ \mathrm{C} & 2.3188040 & -1.6858770 & -1.1303870 \\ \mathrm{C} & 2.6078290 & -0.3171560 & -1.3432330 \\ \mathrm{C} & 2.8904730 & 0.5519300 & -0.2462820 \\ \mathrm{C} & 2.9164650 & -0.0171360 & 1.0698480 \\ \mathrm{H} & 2.5495050 & -1.7923500 & 2.2859870 \\ \mathrm{H} & 2.0362660 & -2.3017130 & -1.9791000 \\ \mathrm{H} & 2.5820210 & 0.0771120 & -2.3531240 \\ \mathrm{H} & 3.1689830 & 0.6128470 & 1.9164350 \\ \mathrm{C} & 1.9267560 & -3.6975960 & 0.3856780 \\ \mathrm{H} & 1.4161630 & -3.8503300 & 1.3411090 \\ \mathrm{H} & 2.8428040 & -4.3026420 & 0.4000530 \\ \mathrm{H} & 1.2884690 & -4.0819100 & -0.4151870 \\ \mathrm{C} & 3.3064630 & 2.0038350 & -0.4426330 \\ \mathrm{H} & 3.0681280 & 2.5292860 & 0.4907740 \\ \mathrm{C} & 4.8351040 & 2.0736810 & -0.6380680 \\ \mathrm{H} & 5.1631970 & 3.1163920 & -0.7118120 \\ \mathrm{H} & -1.9363830 & 1.9328010 & 2.6519790 \\ \mathrm{H} & 5.1378640 & 1.5583540 & -1.5572110 \\ \mathrm{H} & 5.3687270 & 1.6116050 & 0.1994960 \\ \mathrm{C} & 2.5788760 & 2.7220660 & -1.5867970 \\ \mathrm{H} & 2.8644630 & 3.7793120 & -1.6019240 \\ \mathrm{H} & 1.4915640 & 2.6693920 & -1.4705240 \\ \mathrm{H} & 2.8376140 & 2.3015040 & -2.5649170 \\ \mathrm{C} & -3.9446520 & 0.7314670 & -1.1530040 \\ \mathrm{C} & -3.4972440 & 1.6996140 & -2.0549380 \\ \mathrm{C} & -2.3229260 & 2.4133660 & -1.7876160 \\ \mathrm{C} & -1.5831680 & 2.1737390 & -0.6225890 \\ \mathrm{C} & -2.0285710 & 1.2039320 & 0.2788830 \\ \mathrm{C} & -3.2065980 & 0.5028230 & 0.0067560 \\ \mathrm{H} & -4.8474300 & 0.1579690 & -1.3432780 \\ \mathrm{H} & -4.0576550 & 1.8988910 & -2.9638520 \\ \mathrm{H} & -1.9800450 & 3.1672170 & -2.4912520 \\ \mathrm{H} & -1.4277450 & 0.9175600 & 1.6295150 \\ \mathrm{H} & -0.4722250 & 0.5367930 & 3.2169590 \\ \mathrm{H} & 2.7462050 & -0.4189750 \\ \mathrm{H} & 0.0240680 & 3.3367840 \\ \mathrm{H} & & \\ \mathrm{H} & & \end{array}$

$\mathrm{H}$

$$
\mathrm{H}
$$

$-2.9818730$

2.1849240

2.4587720

$-1.3477220$

2.8547830

2.5677580

$\mathrm{H} \quad-1.8471380$

1.5667630

3.6759290

C $\quad-3.5027270$

$-0.5146570$

1.0508970

O -2.6498460

$-0.4489780$

2.0379090

O $\quad-4.4347040$

$-1.3319360$

0.9839760

C $\quad-2.3014680$

$-2.3356230$

$-2.3450830$

H $\quad-2.7788320$

$-1.8536720$

$-3.2005400$

H $\quad-3.0371090$

$-2.5742770$

$-1.5767000$

H $\quad-1.8373270$

$-3.2656990$

$-2.6950920$

C $\quad-1.2306490$

$-1.4637490$

$-1.7688190$

O $\quad-0.9755200$

$-1.4059790$

$-0.5639820$

O $\quad-0.5445300$

$-0.7662710$

$-2.6717970$

$\mathrm{H}$

0.1667210

$-0.2777060$

$-2.1996660$

\section{Y5'}

$\mathrm{Ru} \quad-0.9756380 \quad-0.5734500$

0.1300400

C $\quad-2.5866350$

$-1.7474240$

$-0.6503310$

C $\quad-2.7091900$

$-1.9174380$

0.7717020

C -2.9039310

$-0.7532580$

1.5506840

C $\quad-3.0464390$

0.5327210

0.9494840

C $\quad-2.9502970$

0.7244160

$-0.4406010$

C $\quad-2.6360740$

$-0.4391070$

$-1.2329070$

$\mathrm{H} \quad-2.4602390$

$-2.6204470$

$-1.2827240$

H $\quad-2.9364950$

$-0.8370430$

2.6327340

H -3.1789950

1.3879010

1.6039900

H $\quad-2.5692880$

$-0.3370020$

$-2.3130250$

C $\quad-2.7044740$

$-3.2900930$

1.3932980

H $\quad-2.0558670$

$-3.9772680$

0.8426280

H $\quad-3.7180920$

$-3.7131640$

1.3840010

H -2.3688610

$-3.2544410$

2.4340210

C $\quad-3.2405070$

2.0477840

$-1.1354710$

$\mathrm{H} \quad-2.5800910$

2.0996590

$-2.0115470$

C $\quad-4.6933820$

2.0472580

$-1.6573490$

H -4.8975610

2.9612290

$-2.2268450$

H $\quad-5.4061220$

2.0012630

$-0.8252690$

H $\quad-4.8850940$

1.1917970

$-2.3138260$

C $\quad-2.9828920$

3.2918180

$-0.2758450$

$\mathrm{H} \quad-3.1418980$

4.1954070

$-0.8743580$

$\mathrm{H} \quad-1.9572800$

3.3192450

0.1047220

$\mathrm{H} \quad-3.6668990$

3.3453000

0.5788480

C 4.3293110

1.2683070

0.1808480

C $\quad 4.7008560$

1.6694360

$-1.1013510$

C 4.1574920

1.0294070 


\begin{tabular}{|c|c|c|c|c|c|c|c|}
\hline $\mathrm{C}$ & 3.2361810 & -0.0161860 & -2.1036410 & $\mathrm{H}$ & 0.9104720 & 53840 & 600 \\
\hline $\mathrm{C}$ & 2.8543160 & -0.4099690 & -0.8209270 & $\mathrm{C}$ & 3.1384770 & 1.9277450 & 0.0619050 \\
\hline $\mathrm{C}$ & 3.4057670 & 0.2281750 & 0.2904230 & $\mathrm{H}$ & 2.3015100 & 2.5721270 & -0.2396960 \\
\hline $\mathrm{H}$ & 4.7420490 & 1.7393310 & 1.0680860 & $\mathrm{C}$ & 3.8054030 & 2.5837120 & 1.2891360 \\
\hline $\mathrm{H}$ & 5.4161480 & 2.4754160 & -1.2344550 & $\mathrm{H}$ & 4.1727860 & 3.5836140 & 1.0337730 \\
\hline $\mathrm{H}$ & 4.4634760 & 1.3502020 & -3.2189270 & $\mathrm{H}$ & 4.6573430 & 1.9873140 & 1.6360930 \\
\hline $\mathrm{H}$ & 2.8411860 & -0.4999280 & -2.9907260 & $\mathrm{H}$ & 3.1026940 & 2.6876760 & 2.1228600 \\
\hline $\mathrm{C}$ & 1.9061970 & -1.5018480 & -0.3481760 & $\mathrm{C}$ & 4.1163530 & 1.8408990 & -1.1152180 \\
\hline $\mathrm{C}$ & 0.4463420 & -1.3592200 & -0.8401260 & $\mathrm{H}$ & 4.4443090 & 2.8456640 & -1.4011630 \\
\hline $\mathrm{C}$ & 0.2655660 & -2.0577120 & -2.1851590 & $\mathrm{H}$ & 3.6527280 & 1.3774140 & -1.9929080 \\
\hline $\mathrm{H}$ & -0.6667260 & -1.7600790 & -2.6684650 & $\mathrm{H}$ & 5.0133590 & 1.2655440 & -0.8594540 \\
\hline $\mathrm{H}$ & 0.2432030 & -3.1525680 & -2.0799830 & $\mathrm{C}$ & -2.5405760 & 2.6523320 & 0.2525300 \\
\hline $\mathrm{H}$ & 1.0780140 & -1.8401890 & -2.8921830 & $\mathrm{C}$ & -3.6169510 & 2.7573740 & 1.1098160 \\
\hline $\mathrm{C}$ & 2.5050400 & -2.8996160 & -0.6028000 & $\mathrm{C}$ & -4.3203850 & 1.5801540 & 1.4640590 \\
\hline $\mathrm{H}$ & 3.4807410 & -2.9708550 & -0.1126200 & $\mathrm{C}$ & -3.9340110 & 0.3477930 & 0.9837510 \\
\hline $\mathrm{H}$ & 2.6449340 & -3.0825280 & -1.6698270 & $\mathrm{C}$ & -2.7940160 & 0.1850080 & 0.1271790 \\
\hline $\mathrm{H}$ & 1.8491680 & -3.6745830 & -0.1943940 & $\mathrm{C}$ & -2.1066200 & 1.4007240 & -0.2485100 \\
\hline $\mathrm{C}$ & 2.8495430 & -0.3897880 & 1.5019100 & $\mathrm{H}$ & -2.0032740 & 3.5411760 & -0.0649430 \\
\hline $\mathrm{O}$ & 1.9677890 & -1.3454930 & 1.1267060 & $\mathrm{H}$ & -3.9271280 & 3.7231900 & 1.4972130 \\
\hline $\mathrm{O}$ & 3.0817400 & -0.1488970 & 2.6770150 & $\mathrm{H}$ & -5.1772100 & 1.6471270 & 2.1309020 \\
\hline $\mathrm{C}$ & 1.2057910 & 2.9978620 & 2.0259470 & $\mathrm{H}$ & -4.4920770 & -0.5332700 & 1.2833190 \\
\hline $\mathrm{H}$ & 1.9765030 & 3.5704440 & 1.5058350 & $\mathrm{C}$ & -2.4223640 & -1.0814840 & -0.3862630 \\
\hline $\mathrm{H}$ & 1.6138730 & 2.5275220 & 2.9207710 & $\mathrm{C}$ & -1.1543500 & -1.2390540 & -0.9937950 \\
\hline $\mathrm{H}$ & 0.4082860 & 3.6909000 & 2.3195470 & $\mathrm{C}$ & -0.8404340 & -2.3160970 & -2.0099860 \\
\hline $\mathrm{C}$ & 0.6200320 & 1.9572290 & 1.1246340 & $\mathrm{H}$ & 0.2250210 & -2.2974830 & -2.3086700 \\
\hline $\mathrm{O}$ & 0.2488420 & 0.8464030 & 1.5196480 & $\mathrm{H}$ & -1.4224730 & -2.2007330 & -2.9325020 \\
\hline $\mathrm{O}$ & 0.5042230 & 2.3281960 & -0.1461010 & $\mathrm{H}$ & -1.0280140 & 39830 & 36700 \\
\hline $\mathrm{H}$ & 0.06 & 1.5780770 & -0.6299230 & $\mathrm{C}$ & -3.3772950 & -2.2576810 & -0.2941060 \\
\hline \multirow{2}{*}{\multicolumn{2}{|c|}{ Yts4 }} & & & $\mathrm{H}$ & -4.4081420 & -1.9635260 & -0.5292640 \\
\hline & & & & $\mathrm{H}$ & -3.4035900 & -2.7158050 & 0.7061570 \\
\hline $\mathrm{Ru}$ & 0.7554140 & -0.7938630 & -0.4172970 & $\mathrm{H}$ & -3.1090650 & -3.0539300 & -0.9926450 \\
\hline $\mathrm{C}$ & 0.6898700 & -0.5876140 & 1.7334600 & $\mathrm{C}$ & -1.0837640 & 1.4190130 & -1.2771000 \\
\hline $\mathrm{C}$ & 1.2843700 & -1.8555100 & 1.4653900 & $\mathrm{O}$ & -0.6349300 & 0.2160340 & -1.7612420 \\
\hline $\mathrm{C}$ & 2.4034000 & -1.8504390 & 0.5812290 & $\mathrm{O}$ & -0.6128570 & 2.4378570 & -1.8048600 \\
\hline $\mathrm{C}$ & 3.0906850 & -0.6469180 & 0.1846390 & \multirow{2}{*}{ Y6 } & & & \\
\hline $\mathrm{C}$ & 2.5318540 & 0.5959960 & 0.4673450 & & & & \\
\hline $\mathrm{C}$ & 1.2528800 & 0.5916980 & 1.1517540 & $\mathrm{C}$ & 1.1319560 & 2.2322500 & 0.5591650 \\
\hline $\mathrm{H}$ & -0.2159020 & -0.5204930 & 2.3275000 & $\mathrm{C}$ & 1.9831480 & 1.1032210 & 0.7823290 \\
\hline $\mathrm{H}$ & 2.8002920 & -2.8047000 & 0.2465210 & $\mathrm{C}$ & 2.2623230 & 0.2051420 & -0.2727820 \\
\hline $\mathrm{H}$ & 3.9906110 & -0.7345370 & -0.4135060 & $\mathrm{C}$ & 1.6685870 & 0.4770710 & -1.5490790 \\
\hline $\mathrm{H}$ & 0.7424350 & 1.5384050 & 1.3023090 & $\mathrm{C}$ & 0.8895500 & 1.6306290 & -1.7904730 \\
\hline $\mathrm{C}$ & 0.7230760 & -3.1364610 & 2.0202610 & $\mathrm{C}$ & 0.5983370 & 2.5330080 & -0.7301640 \\
\hline $\mathrm{H}$ & -0.3545280 & -3.0600170 & 2.1928250 & $\mathrm{H}$ & 0.9032700 & 2.8909280 & 1.3908760 \\
\hline $\mathrm{H}$ & 1.1983240 & -3.3736760 & 2.9806230 & $\mathrm{H}$ & 2.3884300 & 0.9364110 & 1.7736810 \\
\hline
\end{tabular}




\begin{tabular}{|c|c|c|c|c|c|c|c|}
\hline$H$ & 8530 & & & $\mathrm{C}$ & & & \\
\hline $\mathrm{H}$ & 0.4726400 & 8029770 & -2.7778940 & $\mathrm{C}$ & 2.2865130 & 1.9096330 & 00156 \\
\hline $\mathrm{Ru}$ & -0.0424760 & 4530300 & 0022920 & C & 2.8536510 & 0.6845930 & 0.5026700 \\
\hline $\mathrm{C}$ & -1.3217870 & 2836030 & 6656520 & $\mathrm{C}$ & 2.7406490 & -0.5119200 & 0.244691 \\
\hline C & $-2.1<$ & 9200 & 00 & $\mathrm{C}$ & 320 & 2910 & 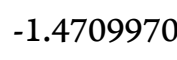 \\
\hline$\sigma_{-1}$ & -1.9 & 5939780 & & $H$ & $0.8 \mathrm{~s}$ & 80 & 015 \\
\hline C & -2.5528270 & -0.6873630 & 30 & $\mathrm{H}$ & 2.38 & 960 & 8220 \\
\hline C & -0.9955570 & -1.63 & & $\mathrm{H}$ & 20 & 80 & 810 \\
\hline & -2.16 & -1.6602240 & -2.649 & $\mathrm{H}$ & 1.83 & 510 & \\
\hline $\mathrm{H}$ & -3.32 & 0.0218090 & -2.04 & $\mathrm{C}$ & 1.1012020 & 290 & -1 . \\
\hline C & -0.6154550 & -2.6201520 & -1.0424540 & $\mathrm{H}$ & 0.1726020 & 3.1246090 & -2.364300 \\
\hline C & -1.1578850 & -2.6184240 & -2.2 & $\mathrm{H}$ & 1.8425990 & 3.6927360 & -2 \\
\hline $\mathrm{H}$ & -2.62 & -1.7 & & $\mathrm{H}$ & 0.92 & 030 & \\
\hline $\mathrm{H}$ & 0.0826310 & -3.3959470 & -0.7421730 & $\mathrm{C}$ & 3.3210870 & -1.8400270 & 0.2218980 \\
\hline $\mathrm{H}$ & -0.8627870 & -3.3720760 & -3.0208750 & $\mathrm{H}$ & 2.6649990 & -2.6235260 & -0.178862 \\
\hline C & -0.9098290 & -2.017 & 1.36 & $\mathrm{C}$ & 4.7 & -2.0 & \\
\hline $\mathrm{O}$ & -0.5491400 & -3.1 & & $\mathrm{H}$ & 120 & 12060 & -0 . \\
\hline $\mathrm{O}$ & -1.3080260 & -1.0739990 & 2.2393160 & $\mathrm{H}$ & 5.4240180 & -1.2872670 & -0.03034 \\
\hline C & -3.1361470 & 1.5854210 & 0.2763240 & $\mathrm{H}$ & 4.6928890 & -1.9912540 & $-1.4 \varepsilon$ \\
\hline $\mathrm{H}$ & -3.18 & 1.90 & & $\mathrm{C}$ & & & -30 \\
\hline $\mathrm{H}$ & -4.1434440 & 1.2641910 & 50 & $\mathrm{H}$ & 3.6938390 & -3.0178290 & 2.00 \\
\hline $\mathrm{H}$ & -2.8838310 & 2.4615620 & 0.8781850 & $\mathrm{H}$ & 2.374 & -1.8527220 & 2.1952860 \\
\hline $\mathrm{C}$ & -1.3496770 & 1.2334590 & 2.833 & $\mathrm{H}$ & 4.0630950 & -1.3065610 & 7490 \\
\hline $\mathrm{H}$ & -0.4736810 & 1.08 & 3 & $\mathrm{C}$ & 100 & -2.0 & \\
\hline $\mathrm{H}$ & -1.3454090 & 2.2724690 & 2.49 & $\mathrm{C}$ & -4.5641100 & -1.4 & -1 \\
\hline $\mathrm{H}$ & -2.2445540 & 1.0782330 & 3.4530260 & $\mathrm{C}$ & -4.9587360 & -0.2792080 & -0.8914020 \\
\hline $\mathrm{C}$ & -0.2 & 3.783 & -0 . & $\mathrm{C}$ & & & 7800 \\
\hline $\mathrm{H}$ & -0.73 & 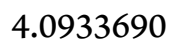 & & 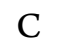 & & & \\
\hline $\mathrm{H}$ & 0.4583900 & 4.6082180 & -1.2 & $\mathrm{C}$ & -2.4799560 & -1.37 & -0.303349 \\
\hline $\mathrm{H}$ & -0.9319170 & 3.6464690 & -1.7 & $\mathrm{H}$ & -2.9968320 & -2 & 655 \\
\hline $\mathrm{C}$ & 3.1896350 & 0.9933220 & & $\mathrm{H}$ & -5.2285030 & & \\
\hline $\mathrm{H}$ & 2.857 & 1.7386790 & -0.8597170 & $\mathrm{H}$ & -5.9303980 & & -1.118 \\
\hline $\mathrm{C}$ & 3.1609230 & -1.6542670 & 1.2583810 & $\mathrm{H}$ & -4.4422820 & 1.2925530 & 0.4924250 \\
\hline $\mathrm{H}$ & 2.1500300 & -1.9700070 & 1.5354370 & $\mathrm{C}$ & -1.9292870 & 0.5233160 & 1.2562800 \\
\hline 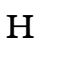 & 3.7997040 & -2.5441240 & & $C_{1}$ & -0.9409440 & & 3720 \\
\hline $\mathrm{H}$ & 3.5390650 & -0.9866150 & 2.0405300 & $\mathrm{C}$ & -1.1359860 & 2.9100280 & 1.1257140 \\
\hline $\mathrm{C}$ & 4.6252220 & -0.5765920 & -0.5073890 & $\mathrm{H}$ & -0.2325820 & 3.4925920 & 0.9333410 \\
\hline $\mathrm{H}$ & 5.2940080 & -1.4442430 & -0.4877690 & $\mathrm{H}$ & -1.4021800 & 3.0440630 & 2.1833900 \\
\hline $\mathrm{H}$ & 4.6642370 & -0.1452570 & -1.5135020 & $\mathrm{H}$ & -1.9509310 & 3.3587090 & 0.5391900 \\
\hline $\mathrm{H}$ & 5.0176310 & 120 & 0.1956370 & $\mathrm{C}$ & -2.4029940 & 0.5604880 & 2.6823110 \\
\hline \multirow{2}{*}{\multicolumn{2}{|c|}{ Yts5 }} & & & $\mathrm{H}$ & -2.6054540 & -0.4585760 & 3.0288310 \\
\hline & & & & $\mathrm{H}$ & -3.3549890 & 1.1066970 & 2.7407340 \\
\hline $\mathrm{Ru}$ & 0.6058230 & 0.5420930 & 0.0874390 & $\mathrm{H}$ & -1.6785610 & 1.0363830 & 3.3454620 \\
\hline & 1.4492900 & 0.7408910 & -1.9613270 & $\mathrm{C}$ & -1.1466920 & -1.8880340 & 0.1103660 \\
\hline
\end{tabular}




\begin{tabular}{|c|c|c|c|c|c|c|c|}
\hline $\mathrm{O}$ & -0.5791950 & -1.0967290 & 0.9962880 & $\mathrm{C}$ & 1.3713730 & 3.1417970 & -1.0 \\
\hline $\mathrm{O}$ & -0.6515990 & -2.9415360 & -0.3057940 & $\mathrm{H}$ & 0.3344130 & 2.7894450 & -1.0419170 \\
\hline \multirow{2}{*}{$\mathbf{Y}$} & & & & $\mathrm{H}$ & 1.3588040 & 4.2346640 & -0.9501630 \\
\hline & & & & $\mathrm{H}$ & 1.8311130 & 2.8888390 & -1.9891800 \\
\hline $\mathrm{C}$ & 2.4173810 & -1.2100650 & -0.8960730 & $\mathrm{C}$ & 3.6015000 & 3.0577140 & 0.1716910 \\
\hline C & 2.3220850 & 0.2161670 & -0.9841520 & $\mathrm{H}$ & 3.6218700 & 4.1527840 & 0.2091620 \\
\hline C & 2.1437810 & 1.0201230 & 0.1938690 & $\mathrm{H}$ & 4.1484940 & 2.6798710 & 1.0423060 \\
\hline $\mathrm{C}$ & 2.0650440 & 0.3385330 & 1.4359960 & $\mathrm{H}$ & 4.1411310 & 2.7419190 & -0.7291770 \\
\hline $\mathrm{C}$ & 2.1620850 & -1.0720370 & 1.5274270 & \multirow{2}{*}{\multicolumn{2}{|c|}{ Ytse }} & & \\
\hline $\mathrm{C}$ & 2.3256000 & -1.8788040 & 0.3721890 & & & & \\
\hline $\mathrm{H}$ & 2.5781980 & -1.7935420 & -1.7979750 & $\mathrm{C}$ & 1.9740310 & 1.9427860 & 0.4720330 \\
\hline $\mathrm{H}$ & 2.4143110 & 0.6905800 & -1.9555120 & $\mathrm{C}$ & 2.7355860 & 0.7313010 & 0.5180400 \\
\hline $\mathrm{H}$ & 1.8832110 & 0.9130020 & 2.3398230 & $\mathrm{C}$ & 2.6908500 & -0.1690710 & -0.5695770 \\
\hline $\mathrm{H}$ & 2.0461710 & -1.5503170 & 2.4954910 & $\mathrm{C}$ & 1.8965190 & 0.1972750 & -1.7114550 \\
\hline $\mathrm{Ru}$ & 0.5035270 & -0.5803830 & -0.1572380 & $\mathrm{C}$ & 1.2017410 & 1.4142930 & -1.8013170 \\
\hline $\mathrm{C}$ & -0.6860040 & -0.8125270 & -1.5791220 & $\mathrm{C}$ & 1.1928560 & 2.2968010 & -0.6722540 \\
\hline $\mathrm{C}$ & -2.0912010 & -0.6700220 & -1.0372560 & $\mathrm{H}$ & 1.9851620 & 2.6123170 & 1.3265020 \\
\hline $\mathrm{C}$ & -2.0022600 & -0.4697810 & 0.4694490 & $\mathrm{H}$ & 3.3271620 & 0.5087270 & 1.3987210 \\
\hline $\mathrm{C}$ & -1.7052160 & -1.3870440 & 1.4847490 & $\mathrm{H}$ & 1.8065040 & -0.5191490 & -2.5234320 \\
\hline $\mathrm{C}$ & -2.3636820 & 0.846 & 0.7 & $\mathrm{H}$ & 0.6 & 562240 & -2.6887450 \\
\hline $\mathrm{C}$ & -1.7608340 & -0.9279210 & 2.8143480 & $\mathrm{Ru}$ & 0.5136750 & 0.3420770 & 0.1660520 \\
\hline $\mathrm{H}$ & -1.5053380 & -2.4303420 & 1.2659380 & $\mathrm{C}$ & -0.5130880 & -0.0706940 & 1.7097750 \\
\hline $\mathrm{C}$ & -2.4227810 & 1.3036120 & 2.0990950 & $\mathrm{C}$ & -1.4784330 & 0.7174880 & 0.9904560 \\
\hline $\mathrm{C}$ & -2.1084290 & 0.3932520 & 3.1161440 & $\mathrm{C}$ & -2.5323080 & 0.1553140 & 0.1117500 \\
\hline $\mathrm{H}$ & -1.5468370 & -1.6207190 & 3.6230780 & $\mathrm{C}$ & -3.5831490 & 0.9902490 & -0.3338240 \\
\hline $\mathrm{H}$ & -2.7155370 & 2.3251670 & 2.3219790 & $\mathrm{C}$ & -2.5764160 & -1.2035670 & -0.3093430 \\
\hline $\mathrm{H}$ & -2.1543840 & 0.7092890 & 4.1541010 & $\mathrm{C}$ & -4.6095080 & 0.5150690 & -1.1465100 \\
\hline $\mathrm{C}$ & -2.7153790 & 1.5440090 & -0.4626610 & $\mathrm{H}$ & -3.6018020 & 2.0330250 & -0.0400700 \\
\hline $\mathrm{O}$ & -3.0478280 & 2.7078520 & -0.6280890 & $\mathrm{C}$ & -3.6284830 & -1.6715410 & -1.1092420 \\
\hline $\mathrm{O}$ & -2.6315310 & 0.6667940 & -1.4884750 & $\mathrm{C}$ & -4.6453690 & -0.8254620 & -1.5395040 \\
\hline $\mathrm{C}$ & -3.1230220 & -1.7071910 & -1.4760240 & $\mathrm{H}$ & -5.3922130 & 1.1986000 & -1.4657670 \\
\hline $\mathrm{H}$ & -2.7929040 & -2.7061080 & -1.1747570 & $\mathrm{H}$ & -3.6254070 & -2.7185330 & -1.3946940 \\
\hline $\mathrm{H}$ & -4.0933030 & -1.5036390 & -1.0115450 & $\mathrm{H}$ & -5.4486050 & -1.2009920 & -2.1662070 \\
\hline $\mathrm{H}$ & -3.2442920 & -1.6956900 & -2.5625300 & $\mathrm{C}$ & -1.5145410 & -2.1801030 & 0.0514790 \\
\hline $\mathrm{C}$ & -0.5394750 & -1.0090320 & -3.0662720 & $\mathrm{O}$ & -1.6701650 & -3.4002580 & -0.0950400 \\
\hline $\mathrm{H}$ & -0.9618380 & -1.9714060 & -3.3926340 & $\mathrm{O}$ & -0.3778060 & -1.7120740 & 0.5306290 \\
\hline $\mathrm{H}$ & -1.0693350 & -0.2300470 & -3.6351660 & $\mathrm{C}$ & -1.8025390 & 2.0399030 & 1.6891930 \\
\hline $\mathrm{H}$ & 0.5100340 & -0.9916090 & -3.3751800 & $\mathrm{H}$ & -1.8900480 & 2.8667570 & 0.9775980 \\
\hline $\mathrm{C}$ & 2.4933570 & -3.3734110 & 0.4742090 & $\mathrm{H}$ & -2.7465390 & 1.9781250 & 2.2473110 \\
\hline $\mathrm{H}$ & 2.1181100 & -3.8790950 & -0.4203080 & $\mathrm{H}$ & -1.0131610 & 2.3108000 & 2.3958510 \\
\hline $\mathrm{H}$ & 3.5552630 & -3.6328380 & 0.5793890 & $\mathrm{C}$ & -0.4610770 & -0.6220510 & 3.0787390 \\
\hline $\mathrm{H}$ & 1.9671970 & -3.7742460 & 1.3458300 & $\mathrm{H}$ & 0.4338920 & -1.2262970 & 3.2472190 \\
\hline $\mathrm{C}$ & 2.1478760 & 2.5415140 & 0.1537330 & $\mathrm{H}$ & -0.4213730 & 0.2375510 & 3.7638590 \\
\hline $\mathrm{H}$ & 1.6643270 & 2.8820340 & 1.0786830 & $\mathrm{H}$ & -1.3609120 & -1.1953390 & 3.3320420 \\
\hline
\end{tabular}




\begin{tabular}{|c|c|c|c|c|c|c|c|}
\hline $\mathrm{C}$ & 0.4695030 & 6620 & 6030 & $\mathrm{H}$ & -3.2766700 & 960 & 160 \\
\hline $\mathrm{H}$ & 0.2240160 & 3.9847970 & 0.2652700 & $\mathrm{H}$ & -1.8064740 & 0.2553680 & 3.6114000 \\
\hline $\mathrm{H}$ & 1.1072640 & 4.3663890 & -1.2176380 & $\mathrm{C}$ & 0.3687640 & -1.4173670 & 2.8918710 \\
\hline $\mathrm{H}$ & -0.4521210 & 3.5391010 & -1.3177990 & $\mathrm{H}$ & 0.5855170 & -0.5727110 & 3.5596830 \\
\hline $\mathrm{C}$ & 3.4679850 & -1.4772890 & -0.6003560 & $\mathrm{H}$ & -0.2488030 & -2.1327050 & 3.4453700 \\
\hline $\mathrm{H}$ & 2.8829430 & -2.1758710 & -1.2128070 & $\mathrm{H}$ & 1.3226550 & -1.8874770 & 2.6425100 \\
\hline $\mathrm{C}$ & 3.6718310 & -2.1194460 & 0.7782190 & $\mathrm{C}$ & -0.5088380 & 3.8676830 & 0.1410470 \\
\hline $\mathrm{H}$ & 2.7190540 & -2.2696060 & 1.2966430 & $\mathrm{H}$ & -0.9355670 & 3.8550440 & 1.1481460 \\
\hline H & 4.1519930 & -3.0972980 & 0.6660310 & $\mathrm{H}$ & 0.0635950 & 4.7980050 & 0.0376400 \\
\hline $\mathrm{H}$ & 4.3188100 & -1.5116600 & 1.4209130 & $\mathrm{H}$ & -1.3234580 & 3.8939940 & -0.5876640 \\
\hline $\mathrm{C}$ & 4.8197010 & -1.2609030 & -1.3114080 & $\mathrm{C}$ & 3.2372550 & -0.6317640 & -0.8089820 \\
\hline $\mathrm{H}$ & 5.3567920 & -2.2107290 & -1.4119620 & $\mathrm{H}$ & 2.9256730 & -1.1168520 & -1.7421180 \\
\hline $\mathrm{H}$ & 4.6845810 & -0.8439430 & -2.3 & $\mathrm{C}$ & 3.3551330 & 4860 & 0.2685600 \\
\hline $\mathrm{H}$ & 5.4549000 & -0.5718190 & -0.7422180 & $\mathrm{H}$ & 2.3986220 & -2.2226130 & 0.4332940 \\
\hline & & & & $\mathrm{H}$ & 4.0831080 & -2.4729950 & -0.0488340 \\
\hline & & & & $\mathrm{H}$ & 3.7042720 & -1.3136760 & 1.2250020 \\
\hline $\mathrm{C}$ & 1.1661470 & 2.1361620 & 0.9837070 & $\mathrm{C}$ & 4.6003560 & 0.04 & -1.0637680 \\
\hline $\mathrm{C}$ & 2.0632720 & 1.0493540 & 0.7773900 & $\mathrm{H}$ & 5.3463630 & -0.7034940 & -1.3545050 \\
\hline $\mathrm{C}$ & 2.2019740 & 0.4407380 & -0.5083720 & $\mathrm{H}$ & 4.5374110 & 0.7883530 & 49520 \\
\hline $\mathrm{C}$ & 1.361 & 0.9434620 & -1.5377130 & 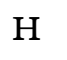 & 0 & 0 & +50 \\
\hline C & 0.4984230 & 2.0603980 & -1.3460400 & \multirow{2}{*}{\multicolumn{2}{|c|}{ Yts8 }} & & \\
\hline $\mathrm{C}$ & 0.3888200 & 2.6821320 & -0.0855060 & & & & \\
\hline $\mathrm{H}$ & 1.0738980 & 2.5628030 & 1.9777340 & $\mathrm{C}$ & -2.4508130 & -1.2034840 & 0.8699250 \\
\hline $\mathrm{H}$ & 2.6424840 & 0.6840890 & 1.6178570 & $\mathrm{C}$ & -2.4224840 & 0.2240970 & 0.8410930 \\
\hline $\mathrm{H}$ & 1.3753560 & 0.4557520 & -2.5074580 & $\mathrm{C}$ & -2.1384070 & 0.9257630 & -0.3794430 \\
\hline $\mathrm{H}$ & -0.1262450 & 2.3949850 & -2.1678100 & $\mathrm{C}$ & -1.9320720 & 0.1500210 & -1.5563200 \\
\hline $\mathrm{Ru}$ & 0.0345730 & 0.3548 & 0.2415 & C & -1 . & 70 & 340 \\
\hline $\mathrm{C}$ & -0.3186080 & -0.9040900 & 1.6798110 & $\mathrm{C}$ & -2.1916540 & -1.9680830 & -0.3178340 \\
\hline $\mathrm{C}$ & -1.6148890 & -0.3673470 & 1.5547650 & $\mathrm{H}$ & -2.6553050 & -1.7193860 & 1.8031480 \\
\hline $\mathrm{C}$ & -2.1220850 & -0.4067490 & 0.1938990 & $\mathrm{H}$ & -2.6105100 & 0.7755730 & 1.7559350 \\
\hline $\mathrm{C}$ & -3.2704380 & 0.3554000 & -0.2128700 & $\mathrm{H}$ & -1.6815560 & 0.6590780 & -2.4822580 \\
\hline $\mathrm{C}$ & -1.3863940 & -1.1714810 & -0.7979370 & $\mathrm{H}$ & -1.7333960 & -1.8174850 & -2.4351040 \\
\hline $\mathrm{C}$ & -3.6254420 & 0.4391840 & -1.5341630 & $\mathrm{Ru}$ & -0.5123740 & -0.5481600 & 0.2095800 \\
\hline $\mathrm{H}$ & -3.8566770 & 0.8687250 & 0.5419680 & $\mathrm{C}$ & 0.5956600 & -0.5825060 & 1.7593700 \\
\hline $\mathrm{C}$ & -1.7863960 & -1.0012790 & -2.1694380 & $\mathrm{C}$ & 1.9327480 & -0.5559800 & 1.2477440 \\
\hline $\mathrm{C}$ & -2.8547150 & -0.2212170 & -2.5311850 & $\mathrm{C}$ & 2.0110800 & -0.5300570 & -0.2650100 \\
\hline $\mathrm{H}$ & -4.4982050 & 1.0177500 & -1.8246780 & $\mathrm{C}$ & 1.8902330 & -1.6467190 & -1.1090250 \\
\hline $\mathrm{H}$ & -1.2483910 & -1.5727490 & -2.9172760 & $\mathrm{C}$ & 2.3108540 & 0.7280090 & -0.8174460 \\
\hline $\mathrm{H}$ & -3.1419760 & -0.1389680 & -3.5756140 & $\mathrm{C}$ & 2.0460380 & -1.4731860 & -2.4927730 \\
\hline $\mathrm{C}$ & -0.6237920 & -2.4780510 & -0.4992530 & $\mathrm{H}$ & 1.7118540 & -2.6343080 & -0.6956230 \\
\hline $\mathrm{O}$ & -0.5710450 & -2.8806960 & 0.7091620 & $\mathrm{C}$ & 2.4599580 & 0.8989910 & -2.1906810 \\
\hline $\mathrm{O}$ & -0.1255270 & -3.0838420 & -1.4759980 & $\mathrm{C}$ & 2.3254910 & -0.2132890 & -3.0288470 \\
\hline$C$ & -2.4148590 & 0.1941750 & 2.7058240 & $\mathrm{H}$ & 1.9554670 & -2.3321640 & -3.1512360 \\
\hline $\mathrm{H}$ & -2.7971430 & 1.1962190 & 2.4894870 & $\mathrm{H}$ & 2.6918490 & 1.8806500 & -2.5930210 \\
\hline
\end{tabular}




\begin{tabular}{|c|c|c|c|c|c|c|c|}
\hline $\mathrm{H}$ & 2.4512370 & -0.1003340 & -4.1018410 & $\mathrm{H}$ & 1.05 & 820 & 460 \\
\hline $\mathrm{C}$ & 2.5229140 & 1.7785570 & 0.2244250 & $\mathrm{C}$ & -4.4956810 & -1.0608010 & 0.9913040 \\
\hline $\mathrm{O}$ & 2.7045220 & 2.9772850 & -0.0446770 & $\mathrm{H}$ & -5.3243070 & -1.5699460 & 0.4896090 \\
\hline $\mathrm{O}$ & 2.5008620 & 1.2792210 & 4239120 & $\mathrm{H}$ & -4.2057600 & .6607600 & 1.8600990 \\
\hline C & 3.0988340 & -1.1840770 & 1.9771980 & $\mathrm{H}$ & -4.8651440 & -0.0956020 & 1.3554780 \\
\hline $\mathrm{H}$ & 2.9947200 & -2.2759160 & 1.9327160 & $\mathrm{C}$ & -3.7556990 & -0.0894690 & -1.2438240 \\
\hline $\mathrm{H}$ & 4.0501330 & -0.9137000 & 1.5120770 & $\mathrm{H}$ & -2.9125200 & 0.0548260 & -1.9227310 \\
\hline $\mathrm{H}$ & 3.1162790 & -0.8909270 & 3.0294280 & $\mathrm{H}$ & -4.5283910 & -0.6559440 & -1.7724150 \\
\hline C & 0.3454930 & -0.6288580 & 3.2434600 & $\mathrm{H}$ & -4.1892860 & 0.8847350 & -0.9899620 \\
\hline $\mathrm{H}$ & 0.7665200 & -1.5356210 & 3.7017950 & $\mathrm{C}$ & 0.9612310 & 2.8410240 & -1.0753690 \\
\hline $\mathrm{H}$ & 0.8076630 & 0.2262130 & 3.7582910 & $\mathrm{O}$ & 1.2829530 & 3.2191120 & 0.0539170 \\
\hline $\mathrm{H}$ & -0.7237620 & -0.6136550 & 3.4742580 & $\mathrm{O}$ & 0.4168970 & 1.6902430 & -1.3824160 \\
\hline $\mathrm{C}$ & -2.2428000 & -3.4733130 & -0.2889670 & $\mathrm{C}$ & 1.1905090 & 3.7182820 & -2.3018020 \\
\hline $\mathrm{H}$ & -1.9281930 & -3.8648740 & 0.6826590 & $\mathrm{H}$ & 1.8543640 & 3.2081300 & -3.0064890 \\
\hline $\mathrm{H}$ & -3.2689180 & -3.8194760 & -0.4691440 & $\mathrm{H}$ & 0.2429840 & 3.8870800 & -2.8220800 \\
\hline $\mathrm{H}$ & -1.6045940 & -3.9083120 & -1.0635220 & $\mathrm{H}$ & 1.6291040 & 4.6716560 & -2.0050970 \\
\hline $\mathrm{C}$ & -2.1658940 & 2.4455280 & 8170 & $\mathrm{C}$ & 1.7363710 & -2.9086370 & -0.0999510 \\
\hline $\mathrm{H}$ & -1.6749250 & 2.7205840 & -1.4009730 & $\mathrm{C}$ & 2.7596230 & -2.8422740 & 0.8347760 \\
\hline $\mathrm{C}$ & -1.4160780 & 3.1506500 & 0.6810900 & $\mathrm{C}$ & 3.4947010 & -1.6531830 & 1.0103270 \\
\hline $\mathrm{H}$ & -0.3619960 & 2.8559670 & 0.7082520 & 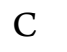 & 3.2113020 & -0.5442280 & 0.22 \\
\hline $\mathrm{H}$ & -1.4586300 & 4.2360620 & 0.5381670 & $\mathrm{C}$ & 2.1614220 & -0.5885930 & -0.7175170 \\
\hline $\mathrm{H}$ & -1.8582510 & 2.9331910 & 1.6597970 & $\mathrm{C}$ & 1.4059630 & -1.7753900 & -0.8697230 \\
\hline $\mathrm{C}$ & -3.6292160 & 2.9268000 & -0.5344520 & $\mathrm{H}$ & 1.1864550 & -3.8288470 & -0.2705080 \\
\hline $\mathrm{H}$ & -3.6687680 & 4.0136720 & -0.6667920 & $\mathrm{H}$ & 3.0093380 & -3.7194160 & 1.4259260 \\
\hline $\mathrm{H}$ & -4.1602690 & 2.4654820 & -1.3741370 & $\mathrm{H}$ & 4.3038340 & -1.6202460 & 1.7341540 \\
\hline $\mathrm{H}$ & -4.1729670 & 2.6801720 & 0.3851740 & $\mathrm{H}$ & 3.8057950 & 0.3601900 & 0.3147480 \\
\hline $\mathbf{A}$ & & & & $\mathrm{C}$ & 0.2584800 & -1.8392980 & -1.8612450 \\
\hline & & & & $\mathrm{O}$ & -0.5369400 & -0.8021480 & -1.7712320 \\
\hline $\mathrm{Ru}$ & -0.1265820 & 0.2354690 & -0.0044070 & $\mathrm{O}$ & 0.1158940 & -2.7977910 & -2.6035790 \\
\hline $\mathrm{C}$ & -1.9236160 & 1.1632940 & 0.7840990 & $\mathrm{H}$ & 2.0390840 & 0.2232010 & -1.4262040 \\
\hline$C$ & -2.1538910 & -0.2468290 & 0.75 & \multirow{2}{*}{\multicolumn{2}{|c|}{ Yts 1-iso }} & & \\
\hline $\mathrm{C}$ & -1.2113870 & -1.0868970 & 1.4197760 & & & & \\
\hline $\mathrm{C}$ & -0.0708560 & -0.5230550 & 2.0483940 & C & -2.1332890 & 1.5567560 & -0.8610890 \\
\hline $\mathrm{C}$ & 0.1237730 & 0.8955510 & 2.1604730 & $\mathrm{C}$ & -2.5880240 & 0.2520460 & -0.6098280 \\
\hline $\mathrm{C}$ & -0.8173000 & 1.7189040 & 1.5065030 & $\mathrm{C}$ & -2.3395960 & -0.3728480 & 0.6584470 \\
\hline $\mathrm{H}$ & -2.5657610 & 1.8264980 & 0.2181620 & $\mathrm{C}$ & -1.6785430 & 0.3725010 & 1.6617720 \\
\hline $\mathrm{H}$ & -1.3039230 & -2.1645670 & 1.3413120 & $\mathrm{C}$ & -1.1826100 & 1.6802900 & 1.3777760 \\
\hline $\mathrm{H}$ & 0.7006860 & -1.1881470 & 2.4245870 & $\mathrm{C}$ & -1.4127860 & 2.3109140 & 0.1250800 \\
\hline $\mathrm{H}$ & -0.6202270 & 2.7814950 & 1.4392890 & $\mathrm{H}$ & -2.2911980 & 1.9926370 & -1.8433240 \\
\hline $\mathrm{C}$ & -3.3222830 & -0.8678880 & 0.0062010 & $\mathrm{H}$ & -3.0940570 & -0.2943740 & -1.3970130 \\
\hline $\mathrm{H}$ & -2.9867510 & -1.8581330 & -0.3237010 & $\mathrm{H}$ & -1.4865980 & -0.0776380 & 2.6302300 \\
\hline $\mathrm{C}$ & 1.3174840 & 1.4801850 & 2.8651740 & $\mathrm{H}$ & -0.6284320 & 2.2141870 & 2.1426690 \\
\hline $\mathrm{H}$ & 2.1397780 & 0.7608850 & 2.9049550 & $\mathrm{Ru}$ & -0.2753840 & 0.3843090 & -0.1582090 \\
\hline U & 1.6540070 & 2.3743910 & 2.3367740 & $\mathrm{C}$ & 0.8306090 & 0.7588190 & -1.9258240 \\
\hline
\end{tabular}




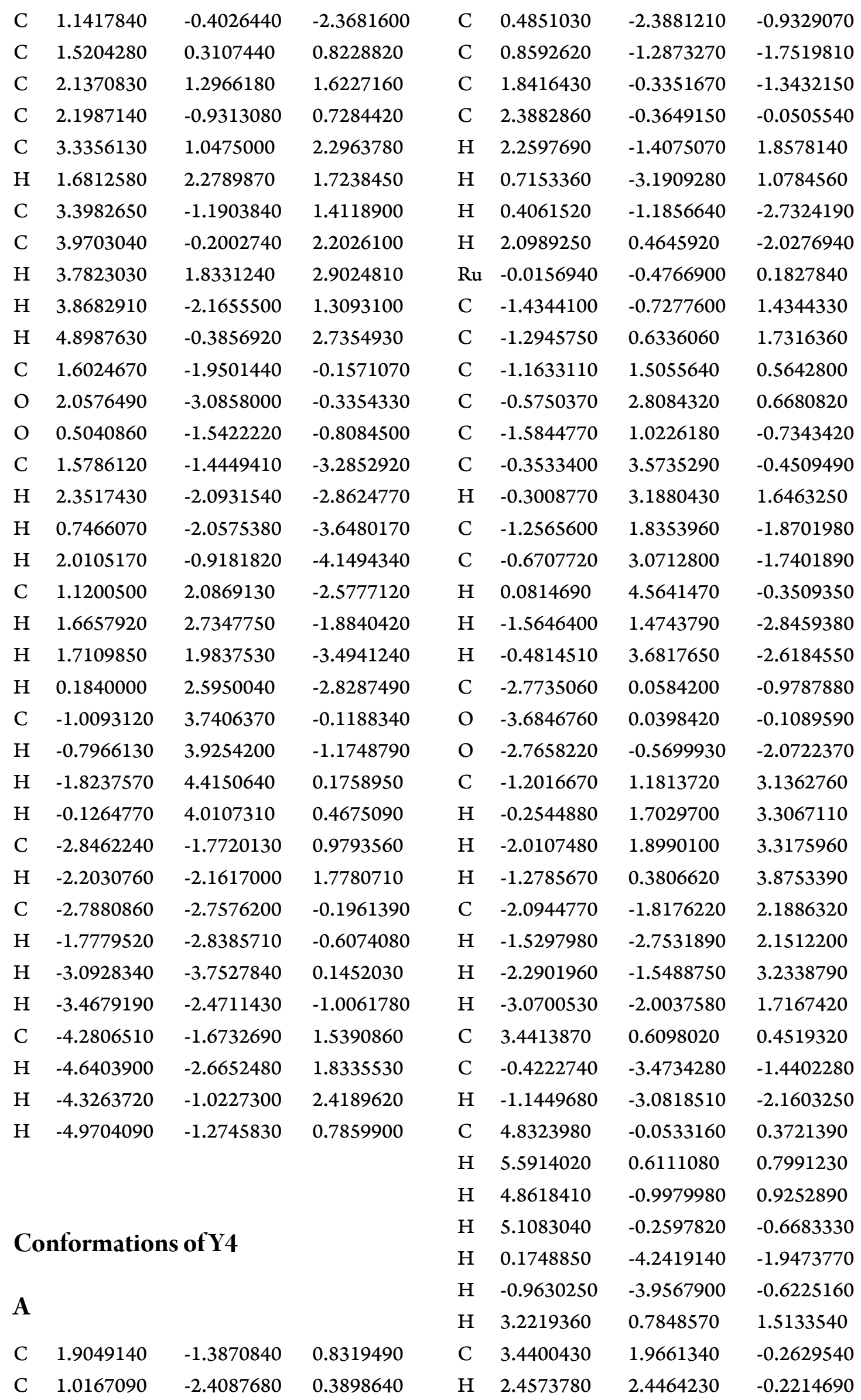




\begin{tabular}{|c|c|c|c|c|c|c|c|}
\hline $\mathrm{H}$ & 0 & 0 & 70 & $\mathrm{C}$ & $-4 . \varepsilon$ & 20 & 0. \\
\hline $\mathrm{H}$ & 3.7329920 & 8745890 & .3148490 & $\mathrm{H}$ & -4.9519360 & 1.1031350 & -0.921285 \\
\hline \multirow{2}{*}{ B } & & & & $\mathrm{H}$ & -5.7655710 & -0.0938990 & 0.1018490 \\
\hline & & & & $\mathrm{H}$ & -4.8863500 & 1.2534110 & 0.8442820 \\
\hline$C$ & -1.8101170 & 1.0905390 & -1.1798610 & $\mathrm{C}$ & -3.6292920 & -1.3693640 & -1.0 \\
\hline C & -2.3879180 & 0.5841860 & 0.0264030 & $\mathrm{H}$ & -2.7209480 & -1.9798200 & -1.110747 \\
\hline$C$ & -1.8043790 & 1.0094760 & .2540230 & $\mathrm{H}$ & -4.4829400 & -2.0403040 & -0.954545 \\
\hline C & -0.7277330 & 9386670 & 0 & $\mathrm{H}$ & 90 & -0.5 & \\
\hline C & -0.2751170 & 5559910 & .0570710 & & & & \\
\hline $\mathrm{C}$ & -0.8175860 & 2.1069810 & -1.1619490 & & & & \\
\hline $\mathrm{H}$ & -2.1749710 & 0.7387950 & -2.1382550 & $\mathrm{C}$ & 0.67 & 370 & -10 \\
\hline $\mathrm{H}$ & -0.27 & 0 & 2.2 & $\mathrm{C}$ & 0. & 2. & -0 \\
\hline $\mathrm{Ru}$ & $-0.1 \varepsilon$ & 0.10 & 0 & $C$ & 60 & 40 & 0670 \\
\hline $\mathrm{C}$ & -0.1641510 & -1.7286760 & 0.5754110 & $\mathrm{C}$ & 1.6095200 & 1.2816550 & 1.0618340 \\
\hline $\mathrm{C}$ & 0.6239660 & -1.9685250 & 870 & $\mathrm{C}$ & 2.186 & 560 & -0.093440 \\
\hline $\mathrm{C}$ & 1.6883460 & -0.9938070 & -0.777 & S & 1.6902630 & 450 & \\
\hline $\mathrm{C}$ & 2.2549080 & -0.7804420 & -2.0772060 & $\mathrm{H}$ & 0.2894670 & 2.3067690 & -2.45759 \\
\hline $\mathrm{C}$ & 2.1462680 & -0.1921250 & 0.3358370 & $\mathrm{H}$ & 1.9229650 & 0.9719950 & 2.05 \\
\hline $\mathrm{C}$ & 3.173 & 0.21 & -2.2 & $\mathrm{Ru}$ & 630 & 60 & \\
\hline $\mathrm{H}$ & 1.9395380 & 730 & -2.9016780 & $\mathrm{C}$ & -2.0 & 0.5763410 & 0.63 \\
\hline $\mathrm{C}$ & 3.0595670 & 0.8735890 & 0.0386690 & $\mathrm{C}$ & -2.3636870 & -0.2154720 & -0.477310 \\
\hline $\mathrm{C}$ & 3.5631960 & 1.073 & -1.2 & $\mathrm{C}$ & 030 & -1.3051480 & -0 . \\
\hline $\mathrm{H}$ & 3.5 & & & $\mathrm{C}$ & & & \\
\hline $\mathrm{H}$ & 3.408 & 4766560 & 0.87 & $\mathrm{C}$ & -0.56 & -1.7700430 & 0.32 \\
\hline $\mathrm{H}$ & 4.2891540 & 1.8614130 & -1.4041870 & $\mathrm{C}$ & -0.33 & -2.8214490 & -2.316872 \\
\hline $\mathrm{C}$ & 2.2030570 & -0.671 & 1.8075950 & $1+$ & -1.9782380 & -1.5644930 & -2 . \\
\hline $\mathrm{O}$ & 2.3156220 & 1.9112940 & 2.00 & $\mathrm{C}$ & 0.4624270 & -2.7054830 & \\
\hline $\mathrm{O}$ & 2.2075060 & 0.2316470 & 2.6881670 & $\mathrm{C}$ & 0.5759250 & -3.2203090 & -1.3 \\
\hline C & 0.3614770 & -3.0725310 & -1.5550460 & $\mathrm{H}$ & -0.2586760 & -3.2518410 & -3.311530 \\
\hline $\mathrm{H}$ & 0.0276040 & -2.6715420 & -2.51 & $\mathrm{H}$ & 1.1 & $-3.0 c$ & 5020 \\
\hline $\mathrm{H}$ & 1.2762940 & -3.6483030 & & $\mathrm{H}$ & & & \\
\hline $\mathrm{H}$ & -0.4090000 & -3.7562490 & -1.1922870 & $\mathrm{C}$ & -0.9458830 & -1.7876350 & 1.8184910 \\
\hline $\mathrm{C}$ & -0.9306600 & -2.6626510 & 1.4314150 & $\mathrm{O}$ & -2.1711920 & -1.8871760 & 2.0949850 \\
\hline $\mathrm{H}$ & -1.90 & -2.26 & & $\mathrm{O}$ & 0.00 & -1.7791930 & 2.6444520 \\
\hline 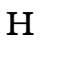 & -1.0571260 & -3.6517170 & .9752870 & $\mathrm{C}$ & -3.5207750 & 0.0695950 & -1.4057000 \\
\hline $\mathrm{H}$ & -0.3552890 & -2.8048230 & 2.3577130 & $\mathrm{H}$ & -3.1842630 & 0.2529870 & -2.4308980 \\
\hline $\mathrm{H}$ & -0.4395400 & 2.4986490 & 2.1005080 & $\mathrm{H}$ & -4.2040920 & -0.7876620 & -1.431714 \\
\hline$\pi$ & -2.1727520 & 0.6094520 & 100100 & $I$ & -4.0824110 & 0.9465900 & -1.07675 \\
\hline $\mathrm{C}$ & 0.7822800 & 3.6225760 & 0.1003590 & $\mathrm{C}$ & -2.9060090 & 1.3871830 & 1.5212900 \\
\hline $\mathrm{H}$ & 0.3038970 & 4.6010320 & 0.2361220 & $\mathrm{H}$ & -2.4168970 & 2.3072260 & 1.8515480 \\
\hline $\mathrm{H}$ & 1.4667740 & 3.4737700 & 0.9390320 & $\mathrm{H}$ & -3.8739030 & 1.6280950 & 1.0644430 \\
\hline $\mathrm{H}$ & 1.3585520 & 3.6590200 & -0.8277020 & $\mathrm{H}$ & -3.1159470 & 0.7855690 & 2.4175770 \\
\hline $\mathrm{C}$ & -3.6010410 & -0.3315650 & 0.0343060 & $\mathrm{H}$ & 2.0511050 & 0.5740410 & -2.2561000 \\
\hline $\mathrm{H}$ & -3.5829460 & -0.8693480 & 0.9896760 & $\mathrm{H}$ & 0.2573490 & 2.7913940 & 1.8443300 \\
\hline
\end{tabular}




$\begin{array}{llll}\mathrm{C} & 3.2879010 & -0.3685140 & -0.0126400 \\ \mathrm{H} & 2.9902690 & -1.1900020 & -0.6774550 \\ \mathrm{C} & -0.7960560 & 3.8817010 & -0.4716540 \\ \mathrm{H} & -0.2478680 & 4.8221220 & -0.6096150 \\ \mathrm{H} & -1.4431050 & 3.7433250 & -1.3420430 \\ \mathrm{H} & -1.4172990 & 3.9876900 & 0.4213000 \\ \mathrm{C} & 4.5994610 & 0.2243490 & -0.5702410 \\ \mathrm{H} & 5.3836190 & -0.5402580 & -0.5698020 \\ \mathrm{H} & 4.4807680 & 0.5847050 & -1.5968600 \\ \mathrm{H} & 4.9444630 & 1.0623440 & 0.0464830 \\ \mathrm{C} & 3.5169080 & -0.9467160 & 1.3890520 \\ \mathrm{H} & 2.5987840 & -1.3432090 & 1.8305860 \\ \mathrm{H} & 4.2443030 & -1.7630450 & 1.3321830 \\ \mathrm{H} & 3.9258380 & -0.1938100 & 2.0728350\end{array}$

